\title{
Modeling Molecules Under Pressure with Gaussian Potentials
}

\author{
Maximilian Scheurer ${ }^{\dagger}{ }^{\text {Andreas Dreuw }},^{\dagger}$ Evgeny Epifanovsky, ${ }^{\ddagger}$ Martin \\ Head-Gordon, ${ }^{\top}, \S$ and Tim Stauch $*, \|, \perp, \#$
}

$\dagger$ †nterdisciplinary Center for Scientific Computing, Heidelberg University, D-69120

Heidelberg, Germany

$\ddagger Q$-Chem Inc., 6601 Owens Dr, Pleasanton, CA 94588, United States of America

IUniversity of California, Berkeley, Pitzer Center for Theoretical Chemistry, South Dr,

Berkeley, CA 94720, United States of America

$\S$ Lawrence Berkeley National Laboratory, Chemical Sciences Division, 1 Cyclotron Rd, Berkeley, CA 94720, United States of America

|l University of Bremen, Institute for Physical and Theoretical Chemistry, Leobener Str. NW2, D-28359 Bremen, Germany

$\perp$ Bremen Center for Computational Materials Science, University of Bremen, Am Fallturm 1, D-28359 Bremen, Germany

\#MAPEX Center for Materials and Processes, University of Bremen, Bibliothekstr. 1, D-28359 Bremen, Germany

E-mail: tstauch@uni-bremen.de

\begin{abstract}
The computational modeling of molecules under high pressure is a growing research area that augments experimental high-pressure chemistry. Here, a new electronic structure method for modeling atoms and molecules under pressure, the Gaussians On Sur-
\end{abstract}


face Tesserae Simulate HYdrostatic Pressure (GOSTSHYP) approach, is introduced. In this method, a set of Gaussian potentials is distributed evenly on the van der Waals surface of the investigated chemical system, leading to a compression of the electron density and the atomic scaffold. Since no parameters other than the pressure need to be specified, GOSTSHYP allows straightforward geometry optimizations and ab initio Molecular Dynamics simulations of chemical systems under pressure for non-expert users. Calculated energies, bond lengths and dipole moments under pressure fall within the range of established computational methods for high-pressure chemistry. A Diels-Alder reaction and the cyclotrimerization of acetylene showcase the ability of GOSTSHYP to model pressure-induced chemical reactions. The connection to mechanochemistry is pointed out.

\section{Introduction}

Throughout the past few decades, the interest in high-pressure chemistry has been remarkable. $^{1-3}$ This enthusiasm is at least partially caused by the availability of diamond-anvil cells ${ }^{4}$ and shock-wave technologies, ${ }^{5}$ which allow the temporary or constant application of extremely high pressure reaching magnitudes of several hundred GPa. Using high-pressure technnologies, structural parameters of molecules ${ }^{6-8}$ and crystals $^{9-12}$ have been found to change under pressure. As a result, many pressure-initiated chemical reactions are known. ${ }^{3,13}$

However, progress in experimental high-pressure chemistry would have been significantly slower if it were not for computational methods that allow the modeling of atoms, molecules and crystals under pressure. ${ }^{14}$ These methods include approaches that place atoms and molecules in soft or hard boxes of various shapes and sizes with confining potentials of variable height. ${ }^{15-24}$ Moreover, by using periodic boundary conditions (PBC) in calculations, the multifaceted behavior of extended systems under high pressure can be predicted, including pressure-induced changes in crystal structures of diverse materials. ${ }^{25-32}$ In many cases, the success of such calculations in terms of comparability with experiments is remarkable. ${ }^{33}$ 
In Molecular Dynamics (MD) simulations, pressure can be applied to the investigated systems by manipulation of the box parameters, ${ }^{34,35}$ allowing the simulation of biological, ${ }^{36}$ organic $^{37}$ and inorganic ${ }^{38-40}$ materials under pressure. In ab initio Molecular Dynamics (AIMD) simulations, ${ }^{41}$ pressure can be applied as well, which has been used, e.g., to simulate liquids, ${ }^{42}$ chemical reactions ${ }^{43,44}$ and spectroscopic properties of molecules ${ }^{45}$ under pressure.

At the electronic-structure level, the application of pressure is far from straightforward, since none of the terms in the electronic Hamiltonian depend on pressure explicitly. The simplest method to apply pressure to molecules is provided by mechanochemical approaches, ${ }^{14}$ in which the compression of the molecules is achieved using mechanical forces. The earliest mechanochemical model of pressure is the Generalized Force-Modified Potential Energy Surface (G-FMPES) approach, ${ }^{46}$ which has its roots in quantum mechanochemistry. ${ }^{47,48}$ In this approach, mechanical forces mimicking pressure pull each atom towards the molecular centroid. The G-FMPES method has been applied to model chemical reactions under pressure ${ }^{49,50}$ and investigate pressure-induced changes in vibrational frequencies. ${ }^{46}$ An extension of the G-FMPES approach, the Hydrostatic Compression Force Field (HCFF), has been proposed recently ${ }^{51}$ and was used to model pressure-induced spin crossover phenomena. In $\mathrm{HCFF}$, the definitions of the surface area and the applied pressure are changed in comparison to G-FMPES. The newest member of the family of mechanochemical methods for the application of pressure to molecules is the eXtended Hydrostatic Compression Force Field (X-HCFF) ${ }^{52}$ in which truly hydrostatic conditions are simulated by pushing each atom inwards strictly perpendicular to the molecular surface. Moreover, in contrast to G-FMPES and $\mathrm{HCFF}$, in $\mathrm{X}-\mathrm{HCFF}$ the user-defined pressure (rather than a guess for this quantity) is applied during a quantum chemical geometry optimization. The applicability of X-HCFF for the accurate reproduction of structural changes in molecules and molecular crystals as well as the simulation of a pressure-induced chemical reaction has been demonstrated. ${ }^{52}$ However, in all mechanochemical methods pressure is applied to the molecule only through a compression of the atomic scaffold, whereas the response of the electron density is not 
modeled directly. Furthermore, the treatment of atoms is precluded by the dependence on the nuclear gradient.

An alternative method for the application of pressure to chemical systems is the $e X$ treme Pressure Polarizable Continuum Model (XP-PCM). ${ }^{53-55}$ In analogy to implicit solvent models, an atom or a molecule is placed inside a cavity. Pressure is subsequently applied by shrinking the cavity size and increasing the Pauli repulsion term of the surrounding medium. The applied pressure is then calculated via a fitting procedure as the negative partial derivative of the electronic energy w.r.t. volume. This ansatz allows the modeling of chemical reactions, ${ }^{54-56}$ spectroscopic properties, ${ }^{57,58}$ as well as electronic ${ }^{59}$ and structural ${ }^{53,60,61}$ changes of chemical systems under pressure.

In this paper, a novel electronic structure method for the simulation of atoms and molecules under pressure, the Gaussians On Surface Tesserae Simulate HYdrostatic Pressure (GOSTSHYP) approach, is introduced. In analogy to XP-PCM, GOSTSHYP considers a chemical system inside a cavity that is confined by a tessellated approximation of the van der Waals surface. Pressure is applied by a dense field of Gaussian potentials located at the tessellation points of the surface, which interact with the electron density. GOSTSHYP solves the shortcomings of previous methods for the simulation of chemical systems under pressure in that 1) the method allows for geometry optimizations and AIMD simulations under user-defined pressures, 2) both atoms and molecules can be subjected to pressure and 3) the compression of electron density due to pressure is modeled realistically. GOSTSHYP is verified by comparing against a range of literature values for energies, geometries, dipole moments and chemical reactions under pressure. The new method has been implemented in Q-Chem ${ }^{62}$ and will be available in a future release of the program package. At present, electronic structure calculations at the levels of Hartree-Fock ${ }^{63,64}$ and Density Functional Theory (DFT) ${ }^{65,66}$ are supported.

When carrying out experiments under high pressure by using a diamond-anvil cell or by compressing molecular crystals mechanically, the observed effects are always a result of a 
combination of the pure influence of pressure on the investigated molecules and pressureinduced changes in the interaction between a molecule and its surrounding, e.g. increased charge-transfer. Hence, experimentally, a separate consideration of these effects is impossible. Even in sophisticated Energy Decomposition Analysis (EDA) schemes, ${ }^{67-69}$ it is far from straightforward to decouple important terms like polarization and charge-transfer from one another when describing such setups. Beneficially, GOSTSHYP allows the separate calculation of the pure influence of pressure on molecules and therefore provides a unique point of view on pressure-induced experiments.

The rest of the paper is structured as follows: After discussing the theoretical background of GOSTSHYP (Section 2) and giving some computational details (Section 3), the method is used to calculate various pressure-dependent quantities and comparisons with literature values are made (Section 4). The paper closes with a conclusion and a road map of planned future developments (Section 5).

\section{Theoretical Background}

In GOSTSHYP, hydrostatic pressure is modeled through a set of Gaussian potentials located at the molecule's van der Waals surface, which is discretized and tessellated through a Lebedev grid. ${ }^{70}$ In this procedure, atom-centered spheres with the scaled (pressure-free) atom-specific van der Waals radii are superimposed and the overlapping regions are omitted, leading to a physically sound tessellation field. Since atomic van der Waals radii are in fact pressure-dependent, ${ }^{71}$ in the following we refer to the scaling factors of the atomcentered spheres as the "tessellation sphere scaling factors". The dependence of the results calculated with GOSTSHYP on the technical parameters of the tessellation routine, i.e. the number of tessellation points per atom and the tessellation sphere scaling factors, is tested in Section 4.1. Each tessera $j$ with the area $A_{j}$ is assigned a Cartesian Gaussian function of 
the form

$$
G_{j}(\boldsymbol{r})=p_{j} \exp \left(-w_{j}\left(\boldsymbol{r}-\boldsymbol{r}_{\boldsymbol{j}}\right)^{2}\right)=p_{j} \tilde{G}_{j}(\boldsymbol{r})
$$

Here, $p_{j}$ is the amplitude of the Gaussian centered at $\boldsymbol{r}_{\boldsymbol{j}}$, whereas $w_{j}$ is referred to as the width parameter. Using the electronic one-particle density matrix $D_{p q}$ and the molecular orbitals $\left\{\phi_{p}\right\}$, the energy penalty by pressure is given by

$$
E_{\mathrm{p}}=\sum_{j} \sum_{p q} D_{p q}\left\langle\phi_{p}\left|G_{j}(\boldsymbol{r})\right| \phi_{q}\right\rangle
$$

This expression includes three-center overlap integrals, in which the mid center, i.e. the Gaussian, corresponds to an s-type function. In the following, determination of the function parameters $w_{j}$ and $p_{j}$ will be outlined. The simplest approximation is that the tessera areas are circular. Making this assumption and acknowledging that not all areas are equal (cf. Figure 1), the radius $\tilde{r}_{j}$ of the circle $j$ that holds the Gaussian $G_{j}$ can be calculated by

$$
\tilde{r}_{j}=\sqrt{\frac{A_{j}}{\pi}} .
$$

The goal of choosing an appropriate width parameter is to create a field of Gaussians that is as smooth and continuous as possible, because a continuous hydrostatic pressure should be modeled. Furthermore, "local high-pressure areas", where Gaussians of the same width and amplitude are densely packed should be avoided (the green points in Figure 1).

Hence, it is reasonable to demand that at the edge of each tessera the amplitude of the accomodated Gaussian reaches half of its maximum value. This way, when neighboring Gaussians that belong to tesserae with similar areas overlap at the border between the two tesserae, their amplitudes add up to the value each of the Gaussian has in the middle of its tessera. Clearly, each Gaussian reaches its maximum when $\boldsymbol{r}=\boldsymbol{r}_{j}$, i.e. at the center of each tessera. At this point, the amplitude is $G_{j, \max }=p_{j}$. Requiring the value of $w_{j}$ such that 

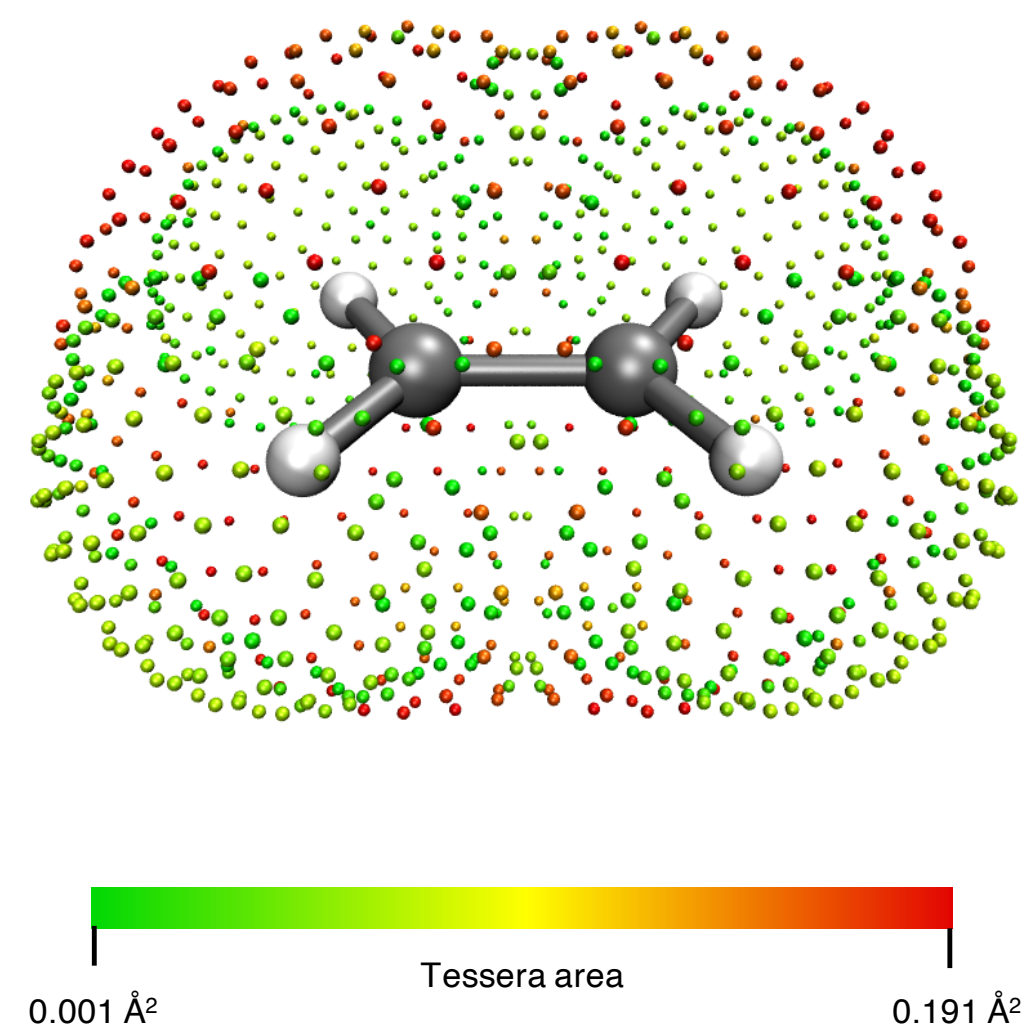

Figure 1: In the employed surface tessellation routine, some tesserae have larger areas than others. In this picture, red signifies large areas, whereas green represents small areas.

$G_{j, \frac{1}{2} \max }=\frac{1}{2} p_{j}$ at the border of the tessera leads to

$$
w_{j}=\frac{\pi \ln 2}{A_{j}} .
$$

The amplitudes $p_{j}$ of the Gaussian functions modeling the pressure potential need to be defined such that the user-defined input pressure is exerted on the embedded molecule. At the interface between the molecule and the surrounding medium, the force acting from the outside $\left(\boldsymbol{F}_{\text {outer }}\right)$, with which the field of Gaussian potentials compresses the electron density, needs to cancel the force acting from the inside $\left(\boldsymbol{F}_{\text {inner }}\right)$, with which the electron density "pushes back". Hence, the requirement is

$$
\boldsymbol{F}_{\text {outer }}=-\boldsymbol{F}_{\text {inner }}
$$


which implies that the absolute values of the force also need to match, i.e.

$$
\left|\boldsymbol{F}_{\text {outer }}\right|=\left|\boldsymbol{F}_{\text {inner }}\right|
$$

or

$$
F_{\text {outer }}=F_{\text {inner }}
$$

Using the definition of pressure,

$$
P=\frac{F_{\perp}}{A}
$$

one realizes that the force acting from the outside is already the normal force $F_{\perp}$. Hence,

$$
F_{\text {outer }}=P_{\text {inp }} A_{j}
$$

where $P_{\text {inp }}$ is the pressure that the user inputs and $\mathrm{A}_{j}$ is the surface area of tessera $j$.

To understand the definition of the force acting from the inside, we imagine taking a Gaussian and displacing along the surface normal vector. Pushing the Gaussian inwards, i.e. closer to the molecule, results in an increase in energy. Pulling it outwards, i.e. away from the molecule, results in a lowering of energy. Hence, the derivative of the electronic energy w.r.t. the position of the Gaussian equals the force with which the molecule pushes the surroundings back. To obtain the force perpendicular to the surface, the scalar product of the derivative vector and the normal vector of the surface $\boldsymbol{n}=\left(n_{x}, n_{y}, n_{z}\right)^{T}$ is used:

$$
\begin{aligned}
F_{\text {inner }} & =\frac{\partial E_{\mathrm{p}}}{\partial x_{j}} n_{x}+\frac{\partial E_{\mathrm{p}}}{\partial y_{j}} n_{y}+\frac{\partial E_{\mathrm{p}}}{\partial z_{j}} n_{z} \\
& =\sum_{p q} D_{p q}\left(\frac{\partial}{\partial x_{j}} n_{x}\left\langle\phi_{p}\left|G_{j}\right| \phi_{q}\right\rangle+\frac{\partial}{\partial y_{j}} n_{y}\left\langle\phi_{p}\left|G_{j}\right| \phi_{q}\right\rangle+\frac{\partial}{\partial z_{j}} n_{z}\left\langle\phi_{p}\left|G_{j}\right| \phi_{q}\right\rangle\right)
\end{aligned}
$$


Inserting eqs 9 and 10 into eq. 6 yields

$$
\begin{aligned}
P_{\mathrm{inp}} A_{j} & =p_{j} \sum_{p q} D_{p q}\left(n_{x} \frac{\partial}{\partial x_{j}}\left\langle\phi_{p}\left|\tilde{G}_{j}\right| \phi_{q}\right\rangle+n_{y} \frac{\partial}{\partial y_{j}}\left\langle\phi_{p}\left|\tilde{G}_{j}\right| \phi_{q}\right\rangle+n_{z} \frac{\partial}{\partial z_{j}}\left\langle\phi_{p}\left|\tilde{G}_{j}\right| \phi_{q}\right\rangle\right) \\
& =-p_{j} \sum_{p q} D_{p q}\left(n_{x}\left\langle\phi_{p}\left|2 w_{j}\left(x-x_{j}\right) \tilde{G}_{j}\right| \phi_{q}\right\rangle+n_{y}\left\langle\phi_{p}\left|2 w_{j}\left(y-y_{j}\right) \tilde{G}_{j}\right| \phi_{q}\right\rangle\right. \\
& \left.+n_{z}\left\langle\phi_{p}\left|2 w_{j}\left(z-z_{j}\right) \tilde{G}_{j}\right| \phi_{q}\right\rangle\right)
\end{aligned}
$$

This expression includes three-center overlap integrals in which the mid center is a p-type function. Rearranging for $\mathrm{p}_{j}$, the amplitude of Gaussian $j$, yields

$$
p_{j}=-\frac{P_{\mathrm{inp}} A_{j}}{\widetilde{F}_{j}},
$$

where

$$
\begin{aligned}
\widetilde{F}_{j} & =\sum_{p q} D_{p q}\left(n_{x}\left\langle\phi_{p}\left|2 w_{j}\left(x-x_{j}\right) \tilde{G}_{j}\right| \phi_{q}\right\rangle+n_{y}\left\langle\phi_{p}\left|2 w_{j}\left(y-y_{j}\right) \tilde{G}_{j}\right| \phi_{q}\right\rangle+n_{z}\left\langle\phi_{p}\left|2 w_{j}\left(z-z_{j}\right) \tilde{G}_{j}\right| \phi_{q}\right\rangle\right) \\
& =\sum_{p q} D_{p q} \mathcal{F}_{j, p q}
\end{aligned}
$$

With the previous expressions at hand, the hydrostatic pressure energy for Gaussian $j$ is given by

$$
\begin{aligned}
E_{\mathrm{p}, j} & =\sum_{p q} D_{p q}\left\langle\phi_{p}\left|G_{j}(\boldsymbol{r})\right| \phi_{q}\right\rangle \\
& =-\sum_{p q} D_{p q}\left\langle\phi_{p}\left|\frac{P_{\mathrm{inp}} A_{j}}{\widetilde{F}} \tilde{G}_{j}\right| \phi_{q}\right\rangle \\
& =-P_{\mathrm{inp}} A_{j} \sum_{p q} D_{p q}\left\langle\phi_{p}\right| \tilde{G}_{j}\left(\boldsymbol{r}\left|\phi_{q}\right\rangle\left(\sum_{p q} D_{p q} \mathcal{F}_{p q}\right)^{-1}\right.
\end{aligned}
$$


The total contribution of the set of Gaussians to the energy, $\mathrm{E}_{\mathrm{p}}$, can be calculated as

$$
E_{\mathrm{p}}=\sum_{j} E_{\mathrm{p}, j},
$$

which entails the computation of three-center overlap integrals. The required integral routines were implemented based on the Obara-Saika scheme ${ }^{72}$ in a development version of the Q-Chem 5.3 program package. ${ }^{62}$

SCF calculations of atoms and molecules in the GOSTSHYP scheme require the contribution to the Fock operator due to the presence of the Gaussian potentials, which reads

$$
\begin{aligned}
V_{j, p q} & =\frac{\partial E_{\mathrm{p}, j}}{\partial D_{p q}}=-P_{\mathrm{inp}} A_{j} \frac{\partial}{\partial D_{p q}} \sum_{r s} D_{r s}\left\langle\phi_{r}\left|\tilde{G}_{j}\right| \phi_{s}\right\rangle\left(\sum_{r s} D_{r s} \mathcal{F}_{j, r s}\right)^{-1} \\
& =\left\langle\phi_{p}\left|G_{j}\right| \phi_{q}\right\rangle+P_{\mathrm{inp}} A_{j}\left[\sum_{r s} D_{r s}\left\langle\phi_{r}\left|\tilde{G}_{j}\right| \phi_{s}\right\rangle\right] \mathcal{F}_{j, p q}\left(\sum_{r s} D_{r s} \mathcal{F}_{j, r s}\right)^{-2}
\end{aligned}
$$

Hence, the Fock operator contribution through the Gaussian potentials is updated in each SCF iteration with the current one-electron density matrix.

For geometry optimizations and AIMD simulations, analytical nuclear gradients were derived and implemented. The explicit derivations and equations can be found in the Appendix. Since the current one-electron density matrix is used for setting up the Gaussian potentials in each SCF step and the tessellation field is re-calculated in each step of a geometry optimization or AIMD simulation, the pressure input by the user, $P_{\text {inp }}$, is applied throughout the entire course of the calculations.

Even for small to medium-sized molecules, thousands of Gaussian potentials are usually considered. In some cases, a small subset of these Gaussian potentials (typically less than 1\%) exhibit negative amplitudes. These are predominantly found at the interface between two van der Waals spheres and lead to an attraction of the electron density in these regions. Since this is an unphysical effect that counteracts the applied pressure, the Gaussian potentials with a negative amplitude are omitted during the SCF procedure and the calculation of the 
gradient.

Although the calculation of three-center overlap integrals using the Obara-Saika scheme is relatively inexpensive from a computational point of view, the large number of Gaussian potentials that interact with each basis function increases the computational cost of GOSTSHYP calculations in comparison to regular, pressure-free geometry optimizations. The magnitude of this relative increase is pointed out for various small to medium-sized molecules throughout the course of this paper. A possible remedy is provided by a screening procedure in which the overlaps between basis functions and Gaussian potentials that exceed a predefined distance are neglected in the calculation of energies and gradients. The implementation of such a procedure as well as its performance in terms of accuracy and saving of computation time will be presented in a future publication.

\section{Computational Methodology}

All calculations were run using a development version of the Q-Chem 5.3 program package $^{62}$ in which the GOSTSHYP approach was implemented. Three-center overlap integrals calculated with the Obara-Saika scheme ${ }^{72}$ were verified by comparing against results generated with Mathematica. ${ }^{73}$ Analytical gradients were verified by comparing against finite differences. The atomic van der Waals radii by Bondi were used. ${ }^{74}$ Various levels of theory, i.e. Hartree-Fock ${ }^{63,64}$ and $\mathrm{DFT}^{65,66}$ with different basis sets, were applied to allow valid comparisons to literature values. The applied electronic structure methods are pointed out throughout the paper.

Atomic and molecular volumes were estimated using a numerical integration scheme. ${ }^{75}$ Cube files for the ground state electron density were generated in Q-Chem using the standard boundaries of the mesh box $( \pm 3 \AA$ around the maximum/minimum of the molecular coordinates) and a grid spacing of $0.1 \AA$. The number of grid points with an electron density larger than 0.001 a.u. ${ }^{75}$ was divided by the number of total grid points and multiplied with 
the box volume yielding the molecular volume. The integration scheme was compared to results from ref. 75 and was found to agree well with their Monte-Carlo technique (data not shown).

Born-Oppenheimer ab initio molecular dynamics (BOMD) simulations of the Diels-Alder reaction between cyclopentadiene and ethylene were run using an integration time step of $20 \mathrm{au}$. Ten independent simulations were run for 1800 time steps at a temperature of 298 K. Initial velocities were randomly generated from a Maxwell-Boltzmann distribution using the given temperature.

\section{Results and Discussion}

\subsection{Dependence on Adjustable Parameters}

The number of tessellation points per atom and the tessellation sphere scaling factors are the only adjustable parameters in the GOSTSHYP model. In this Section, the dependence of calculated structural data on these parameters is tested using the example of trans1,3-butadiene, since reference values calculated with XP-PCM ${ }^{53-55}$ are available. ${ }^{53}$ These calculations were carried out at the B3LYP $76-78 / 6-31 G(d, p)^{79}$ level of theory.

First, the dependence of the length of the carbon-carbon double bond in trans-1,3butadiene on the number of tessellation points per atom with a fixed tessellation sphere scaling factor of 1.2 is tested (Figure $2 \mathrm{a}$ ). When adjusting the number of tessellation points per atom to discrete values between 6 and 590, the bond length decreases smoothly with increasing pressure, which agrees well with chemical intuition. This decrease is most pronounced when using the smallest numbers of tessellation points per atom (6 and 26). At 110 tessellation points per atom, the results are converged, signifying a smooth hydrostatic pressure field created by the Gaussian potentials. To ensure smoothness in all cases, 302 tessellation point per atom are used as a precaution throughout the remainder of the paper, unless noted otherwise. 
a)

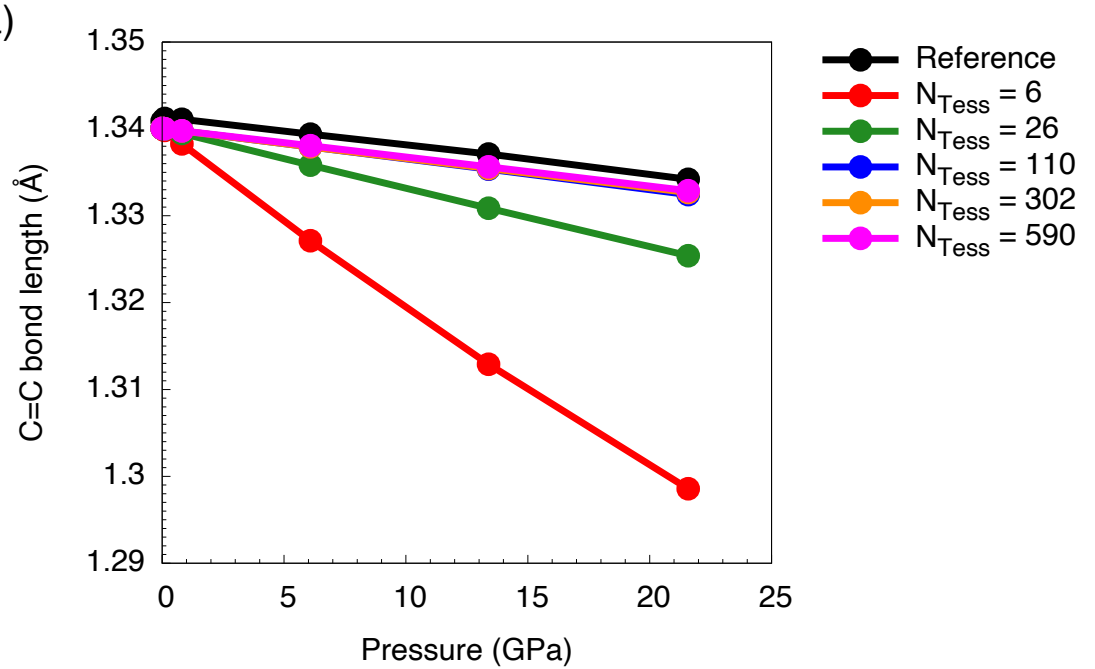

b)

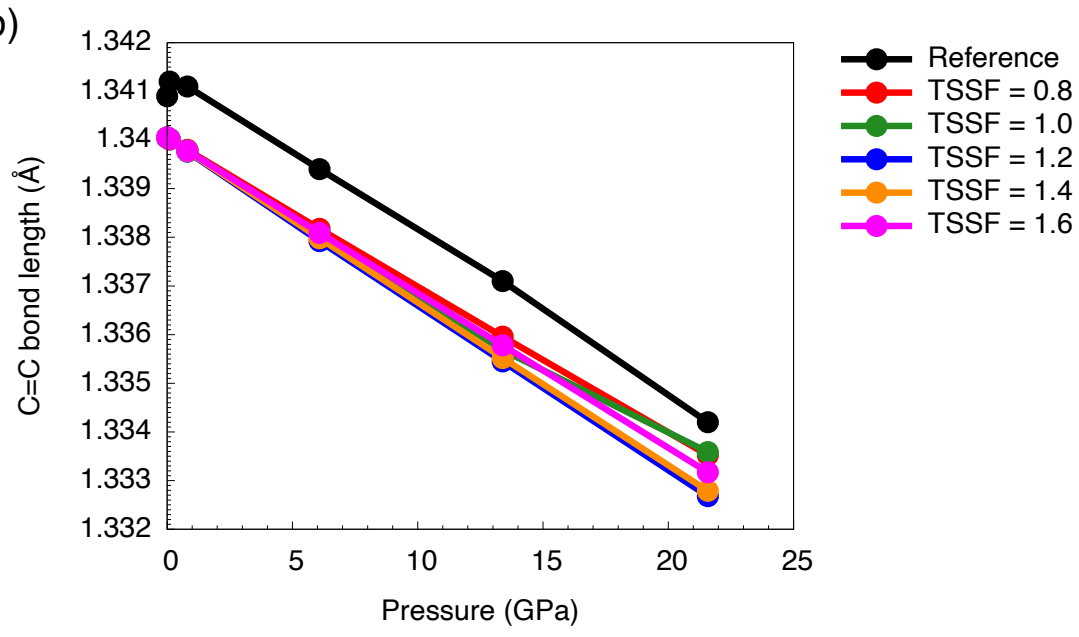

Figure 2: Length of the carbon-carbon double bonds in trans-1,3-butadiene as a function of pressure, applied with the GOSTSHYP model at the B3LYP ${ }^{76-78} / 6-31 \mathrm{G}(\mathrm{d}, \mathrm{p})^{79}$ level of theory. (a) The number of tessellation points per atom $\left(\mathrm{N}_{\text {Tess }}\right)$ was varied and the tessellation sphere scaling factor was set to 1.2. b) The tessellation sphere scaling factor (TSSF) was varied and 302 tessellation points per atom were set. Reference values refer to XP-PCM ${ }^{53-55}$ results at the B3LYP/6-31G(d,p) level of theory and were taken from ref. 53.

Turning to the tessellation sphere scaling factor, it should be noted that, typically, in polarizable continuum models a scaling factor of 1.2 is applied to the atomic van der Waals radii to account for the remaining distance between the solute and the simulated solvent molecules. ${ }^{70}$ By contrast, the atomic van der Waals radii were found to decrease under pressure in an atom-specific manner. ${ }^{71}$ In GOSTSHYP, however, the results for the carboncarbon bond lengths in trans-1,3-butadiene hardly change when adjusting the tessellation 
sphere scaling factors to different values while fixing the number of tessellation points per atom to 302 (Figure 2b). Even at high pressures of over $20 \mathrm{GPa}$, the difference in these bond

lengths is only approx. $10^{-3} \AA$ for scaling factors between 0.8 and 1.6. This independence of the results from the scaling factor is very beneficial, since it eliminates the need to adjust this parameter. In addition, it should be noted that the decrease in the carbon-carbon double bond lengths in trans-1,3-butadiene at low pressures is monotonic and smoother than in XP-PCM. Unless otherwise noted, the typical value for the tessellation sphere scaling factor of 1.2 is used throughout the remainder of the paper, since this value yields roughly the same slope of the carbon-carbon double bond length with increasing pressure as XP-PCM. Note that it may become necessary to increase the tessellation sphere scaling factor for computations on multiple molecules to avoid generation of tessellation points between the individual fragments (cf. Section 4.5).

The independence of the structural data calculated with GOSTSHYP from the two adjustable parameters (the number of tessellation points per atom and the tessellation sphere scaling factor) that was demonstrated here is a very valuable feature of the GOSTSHYP approach, since it allows geometry optimizations of molecules under pressure in a black-box manner by using the default values suggested here and specifying only the desired pressure as an input. This is made possible by the fact that the parameters of the Gaussian, i.e. their amplitudes and widths, react towards changes in the tessellation field, thus guaranteeing that the input pressure is adjusted during the geometry optimization or the AIMD run.

\subsection{Pressure-Dependent Energies and Volumes of Simple Test Sys- tems}

The GOSTSHYP scheme enables the calculation of electronic energies of atoms and molecules as a function of pressure. In this Section, the total electronic energies of the hydrogen atom and the $\mathrm{H}_{2}^{+}$cation calculated with GOSTSHYP at the Hartree-Fock ${ }^{63,64} /$ cc-pVQZ $^{80}$ level of theory are compared to literature values. 
As expected, the total energy of the hydrogen atom increases as a function of pressure (Figure 3a), which is due to the energy contribution by the Gaussian potentials as well as the compression of electron density. Until pressures of approx. $3000 \mathrm{GPa}$, the agreement between GOSTSHYP and literature values, which were generated by placing the hydrogen atom inside boxes with different sizes and impenetrable walls, ${ }^{21}$ is remarkable. At higher pressures, the increase in energy predicted by GOSTSHYP is more pronounced than in the literature. However, pressures of several TPa are irrelevant for applications in chemical laboratories, given that pressures in the range of a few hundred GPa are available experimentally today. ${ }^{4}$ The ability to apply hydrostatic pressure to atoms distinguishes GOSTSHYP from the mechanochemical models of pressure ${ }^{46,51,52}$ which require at least two atoms to work, and emphasizes the connection between GOSTSHYP and XP-PCM ${ }^{53-55}$ which also allows for the treatment of single atoms.

In the case of the $\mathrm{H}_{2}^{+}$cation (Figure 3b), the GOSTSHYP results again agree well with literature values, which were generated by placing the ion inside a spheroidal box ${ }^{18}$ throughout the entire tested pressure range. A geometry optimization step with GOSTSHYP for $\mathrm{H}_{2}^{+}$ at the $\mathrm{HF} / \mathrm{cc}-\mathrm{pVQZ}$ level of theory is on average 8.4 times more expensive in terms of CPU time than a regular, pressure-free geometry optimization step. The observed increase in electronic energy is more pronounced than in the case of the mechanochemical HCFF model, ${ }^{51}$ since the mechanochemical models of pressure omit a reproduction of the compression of electron density and the accompanying increase in energy. Instead, in the mechanochemical models pressure is simulated only via the compression of the atomic scaffold. This important point is expanded on in Section 4.4.

The results presented here demonstrate that the electronic energies calculated with GOSTSHYP are chemically intuitive and fall within the range of the results calculated with established models of pressure. In this regard, GOSTSHYP is clearly superior to simpler mechanochemical models of pressure, since single atoms can be subjected to pressure and the increase in energy due to the compression of electron density is accounted for. Based 
a)

$\mathrm{H}$ atom
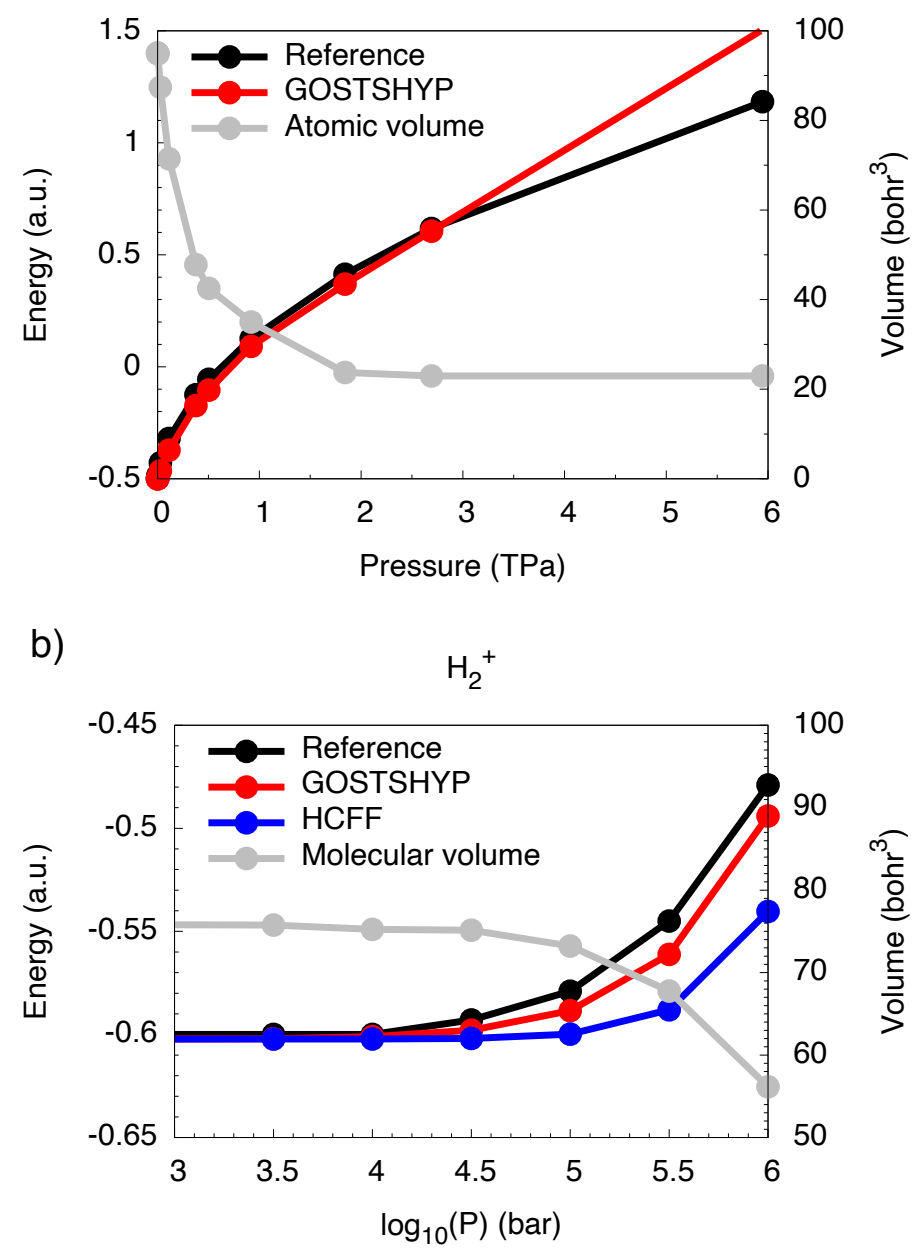

Figure 3: Total electronic energies and volumes of the hydrogen atom (a) and $\mathrm{H}_{2}^{+}$cation (b) under pressure, calculated with GOSTSHYP at the Hartree-Fock ${ }^{63,64} /$ cc-pVQZ $^{80}$ level of theory. Reference values were taken from refs. 21 (a) and 18 (b). HCFF results were taken from ref. 51 .

on the methodology outlined in Section 3, the pressure-dependent volumes of the hydrogen atom and the $\mathrm{H}_{2}^{+}$cation, which are measures of the degree of electron density compression, were calculated using the electron densities and geometries obtained with GOSTSHYP (Figure 3). In both cases, a continuous and marked volume decrease with increasing pressure can be observed, which agrees with chemical intuition. The volume of the hydrogen atom reaches an asymptote of approx. $23 \mathrm{bohr}^{3}$ at $2 \mathrm{TPa}$. Within the smaller pressure range considered for the $\mathrm{H}_{2}^{+}$cation, an asymptotic value has not yet been reached. The volumes 
of more complicated molecules under pressure are discussed in Section 4.6.

\subsection{Structural Parameters Under Pressure}

Crucially, the GOSTSHYP model provides the possibility to perform geometry optimizations under a predefined hydrostatic pressure. While changes in the length of the carbon-carbon double bonds in trans-1,3-butadiene as a function of pressure have already been discussed in Section 4.1 and agreement with literature values was found to be remarkable, in this Section the diborane molecule serves as an additional, independent test system for assessing the accuracy of the structural data under pressure calculated with GOSTSHYP. For this system, reference data calculated with XP-PCM ${ }^{53-55}$ at the M06-2X ${ }^{81} / 6-311++\mathrm{G}(\mathrm{d}, \mathrm{p})^{79}$ level of theory are available. ${ }^{53}$ Therefore, GOSTSHYP calculations were carried out at the same level of theory.

Our focus lies on pressure-induced changes in the distance between the boron atoms, between a boron atom and a bridging hydrogen atom $\left(\mathrm{H}_{\mathrm{b}}\right)$ and between a boron atom and a terminal hydrogen atom $\left(\mathrm{H}_{\mathrm{t}}\right)$ in diborane (Figure 4). As expected, these distances decrease in a fairly linear manner with increasing pressure. Similar effects have been observed before with the mechanochemical X-HCFF model. ${ }^{52}$ Since pressure is proportional to the normal component of the force, this is the expected behavior in the harmonic regime of the compression coordinate. In the case of the boron-boron distance, the XP-PCM results are matched almost perfectly, albeit this quantity decreases more smoothly as a function of pressure in GOSTSHYP. The pressure-induced decrease in the boron-hydrogen distances calculated with GOSTSHYP is less steep than in XP-PCM. It can be assumed that these differences originate from the implicit consideration of the solvent, which is included in XPPCM but neglected in GOSTSHYP. The results show that the boron-boron distance can be compressed easily by pressure and that this coordinate is much softer than the boronhydrogen bonds. This opens up the intriguing perspective of comparing the compressibilities of different coordinates in a molecule. 

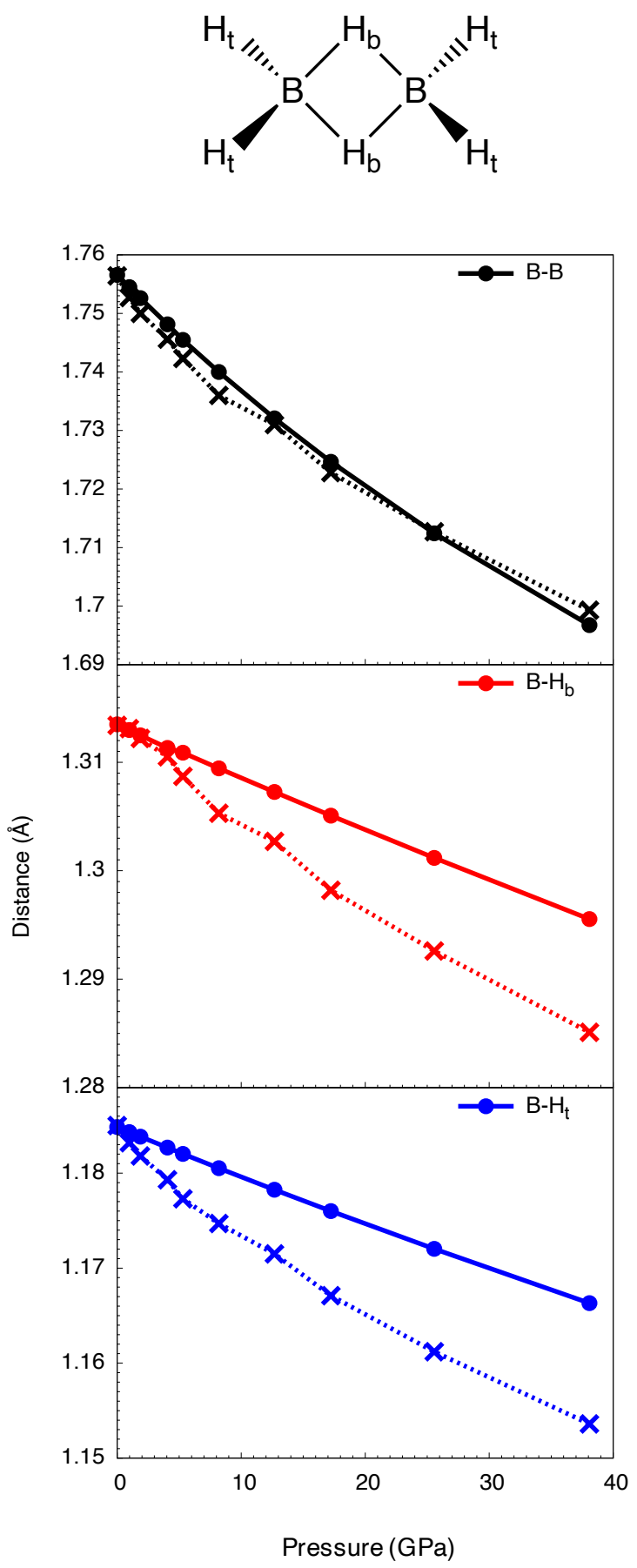

Figure 4: Various distances in the diborane molecule under pressure, calculated with GOSTSHYP at the M06-2X ${ }^{81} / 6-311++\mathrm{G}(\mathrm{d}, \mathrm{p})^{79}$ level of theory (circles connected by solid lines). Reference values calculated with XP-PCM ${ }^{53-55}$ at the same level of theory (crosses connected by dotted lines) were taken from ref. 61 . 


\subsection{Dipole Moment of Water Under Pressure}

To test the suitability of GOSTSHYP to calculate a molecular property that depends both on the pressure and the electron density, the dipole moment of water at different pressures was calculated at the B3LYP $76-78 /$ cc-pVDZ $^{80}$ level of theory, since reference values for this system exist: Cruz and Soullard considered the water molecule in a hard spherical box of varying size and found that a decrease in box size, which simulates an increase in pressure, leads to an initial increase in the dipole moment of water of up to $20 \%$ at a pressure of $1250 \mathrm{GPa}$ and a subsequent decrease. ${ }^{82}$ Kang and co-workers, on the other hand, carried out AIMD simulations of liquid water at elevated temperatures and pressures and calculated the

average dipole moment of water. ${ }^{83}$ It was found that, e.g., the dipole moment increased by approx. $35 \%$ when adjusting the pressure to $51.7 \mathrm{GPa}$ at a temperature of $2800 \mathrm{~K}$.

Geometry optimizations using GOSTSHYP yield a curve for the dipole moment of water under pressure that is qualitatively identical to the one found by Cruz and Soullard, ${ }^{82}$ who also considered a single molecule (Figure 5). The dipole moment of water initially increases with increasing pressure, followed by a gradual decrease. The maximum increase in dipole moment is $17 \%$, which compares favorably with the value of $20 \%$ reported previously. In GOSTSHYP, however, the maximum increase in dipole moment is reached already at approx. $300 \mathrm{GPa}$, i.e. at lower values than in the computations by Cruz and Soullard. At the same time, the pressure-induced increase in the dipole moment of water found with GOSTSHYP is less pronounced than in the study by Kang and co-workers, ${ }^{83}$ presumably because only a single molecule is treated by GOSTSHYP and intermolecular interactions such as polarization as well as temperature effects are neglected. However, as mentioned in Section 1, GOSTSHYP allows the calculation of the influence of pressure on an isolated molecule without the interfering effect of modified interactions with neighboring molecules, which is an important complement to the work reported previously. ${ }^{83}$ In summary, it can be stated that the dipole moment of water calculated with GOSTSHYP at various pressures agrees qualitatively with literature values, and any discrepancies between the values can be 


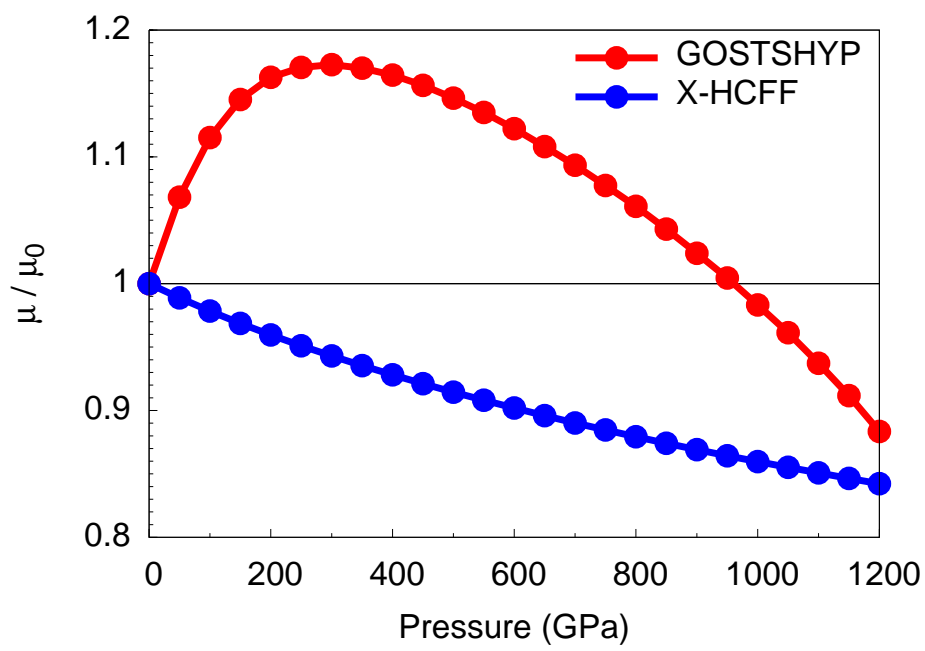

Figure 5: Ratio of the dipole moment of water under pressure and its dipole moment in the pressure-free state, $\mu / \mu_{0}$, calculated with GOSTSHYP and X-HCFF ${ }^{52}$ at the B3LYP $\mathrm{L}^{76-78} / \mathrm{cc}$ $\mathrm{pVDZ}^{80}$ level of theory. The values of the dipole moments are given in the Supporting Information (Table S1).

traced back to the fundamental differences in the underlying models. A geometry optimization step with GOSTSHYP for the water molecule at the B3LYP/cc-pVDZ level of theory is on average 2.8 times more expensive in terms of $\mathrm{CPU}$ time than a pressure-free geometry optimization step.

For comparison, the dipole moment of water at varying pressures was calculated with $\mathrm{X}-\mathrm{HCFF},{ }^{52}$ in which compression is modeled purely mechanically (Figure 5). The observed progression of the dipole moment is qualitatively different to all previously calculated results in that the dipole moment consistently decreases with increasing pressure. The underlying reason is the missing description of the compression of electron density in the mechanochemical models, which leads to qualitatively different results for the structural parameters of the water molecule under pressure (cf. the Supporting Information, Figure S1): The observed decrease in the $\mathrm{O}-\mathrm{H}$ bond length upon application of pressure is much more pronounced in $\mathrm{X}$-HCFF than in GOSTSHYP and, even more crucially, GOSTSHYP predicts a slight decrease in the $\mathrm{H}-\mathrm{O}-\mathrm{H}$ bond angle under pressure, whereas a significant increase is found by $\mathrm{X}$-HCFF. These discrepancies in the structural parameters of water under pressure explain 
the qualitative differences in dipole moment and demonstrate that mechanochemical models are too simplistic to capture the relevant effects. Instead, a more sophisticated treatment of the compression of electron density is needed to reproduce physically sound dipole moments under pressure.

\subsection{Pressure-Induced Chemical Reactions}

The ability of GOSTSHYP to model a pressure-initiated chemical reaction is first demonstrated using the example of a Diels-Alder reaction. The possibility to induce this class of reactions by pressure has been pointed out in the literature. ${ }^{2,49}$ In particular, using XP$\mathrm{PCM}^{53-55}$ it was found that at a pressure of $11.2 \mathrm{GPa}(\mathrm{T}=0 \mathrm{~K})$ the Diels-Alder reaction of cyclopentadiene and ethylene becomes almost barrierless. ${ }^{55}$ It is interesting to investigate whether this behavior can be reproduced by GOSTSHYP. In this Section, a tessellation sphere scaling factor of 1.8 was used to avoid the generation of tessellation points between the fragments, since, when modeling reactions under pressure, it is important to ensure that the entire system is in a single cavity.

The application of pressure to a complex of cyclopetadiene (diene) and ethylene (dienophile) via the static GOSTSHYP approach $(\mathrm{T}=0 \mathrm{~K})$ leads to a monotonic decrease in the distance $d$ between the diene and the dienophile (Figure 6). Initially, $d$ exhibits two small downward steps, which can be traced back to minor conformational changes of the diene relative to the dienophile. Between 39 and $40 \mathrm{GPa}$, a sudden significant decrease of $d$ marks the formation of the reaction product through a pressure-induced Diels-Alder reaction. From $40 \mathrm{GPa}$ onward, the reaction product is compressed only insignificantly.

The pressure required to induce the Diels-Alder reaction between cyclopentadiene and ethylene via GOSTSHYP is by a factor of approx. 3.5 higher than in XP-PCM. This discrepancy can be traced back to the fundamental differences in the computational models. However, it should be pointed out that GOSTSHYP allows geometry optimizations of molecules at a user-defined pressure, which is particularly valuable in the treatment of pressure-induced 

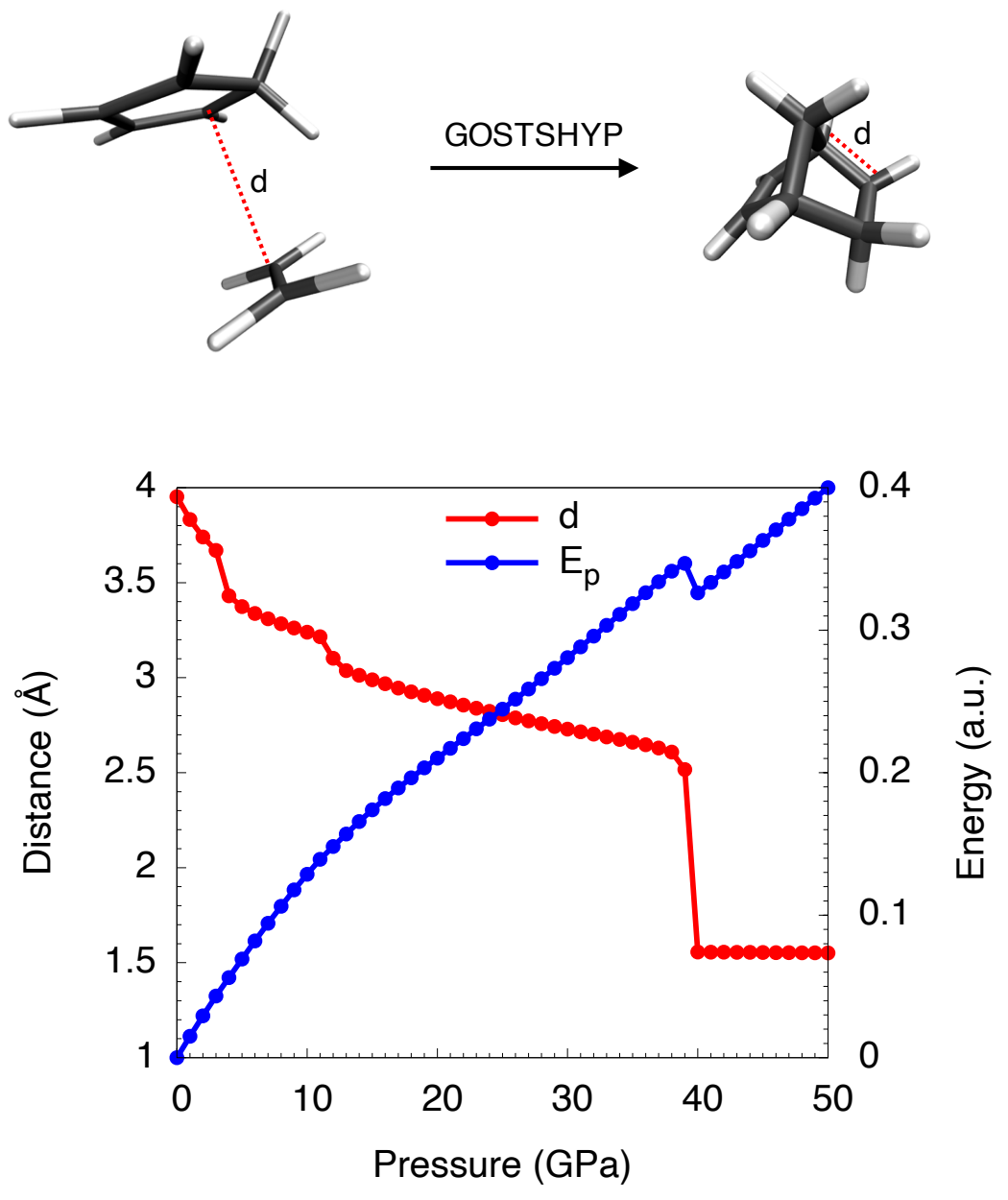

Figure 6: Pressure-induced Diels-Alder reaction of cyclopentadiene and ethylene, modeled with GOSTSHYP at the $\mathrm{PBE}^{84} / \mathrm{cc}^{\mathrm{p}} \mathrm{pDDZ}{ }^{80}$ level of theory. $d$, which refers to the distance between the carbon atoms in cyclopentadiene and ethylene that form a bond during this reaction, is plotted as a function of pressure. The contribution of the pressure to the energy $\left(\mathrm{E}_{\mathrm{p}}\right)$ is plotted as well.

chemical reactions. A geometry optimization step with GOSTSHYP at the PBE/cc-pVDZ level of theory for the system considered here is on average 8.2 times slower in terms of CPU time than a pressure-free geometry optimization step.

The pressure-induced Diels-Alder reaction characterized here can also be understood in terms of the contribution of the Gaussian potentials to the electronic energy, $E_{\mathrm{p}}$ (Eq (2) and Figure 6). Along the reaction coordinate, several regimes in which $\mathrm{E}_{\mathrm{p}}$ increases linearly can be observed. Since pressure is proportional to the normal component of the compressing force, the linear regimes signify harmonicity, whereas the observed kink between 


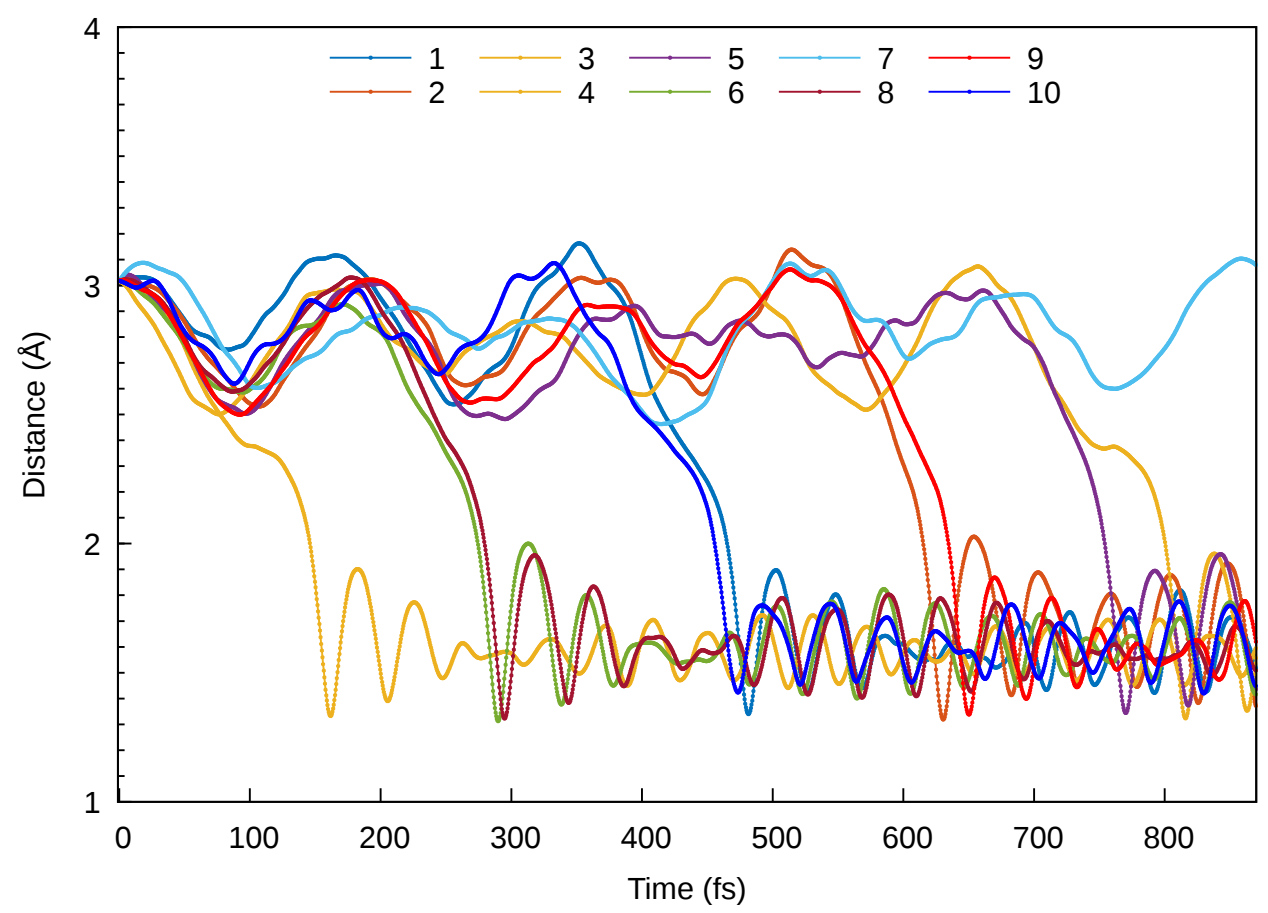

Figure 7: Time-dependent progression of the average distance between the carbon atoms in cyclopentadiene and ethylene that form a bond during the Diels-Alder reaction during the BOMD simulation under a constant pressure of $40 \mathrm{GPa}$, applied via the GOSTSHYP model at the $\mathrm{PBE}^{84} / \mathrm{cc}-\mathrm{pVDZ}{ }^{80}$ level of theory. Each line represents an individual trajectory.

39 and $40 \mathrm{GPa}$ is an instance of anharmonicity due to the profound changes in geometry and electronic structure that accompany the formation of the product of the pressure-induced Diels-Alder reaction. The close connection of pressure and force opens up the interesting possibility of investigating how molecules respond to pressure in terms of strain. ${ }^{47}$ This line of thought is followed in Section 4.6.

Since nuclear gradients can be calculated with GOSTSHYP, it is possible to carry out $a b$ initio Molecular Dynamics (AIMD) simulations. To showcase the capabilities of GOSTSHYP in this regard, as a proof-of-principle Born-Oppenheimer Molecular Dynamics (BOMD) simulations of cyclopentadiene and ethylene under a constant pressure of $40 \mathrm{GPa}$ at $298 \mathrm{~K}$ were carried out (cf. Section 3 for details on the simulation setup). Throughout the ten simulated trajectories, the average distance between the carbon atoms in cyclopentadiene and ethylene that form bonds during the Diels-Alder reaction oscillates visibly (Figure 7), which 
is a result of the compressing influence of the Gaussian potentials and the repulsion of the electron clouds of the molecules. During the simulation time, in nine out of ten trajectories a Diels-Alder reaction can be observed, which is signified by a marked decrease of the average distance between cyclopentadiene and ethylene during bond formation. A movie of a representative trajectory in which the Diels-Alder adduct is formed can be found in the Supporting Information. After carbon-carbon bonds have been formed, the molecule still oscillates, which is again a result of the interplay between compression due to the Gaussian potentials and the restoring force of the molecule. These oscillations occur with an increased frequency as soon as the bonds have been formed, which is due to the higher force constant of the covalent bonds compared to the intermolecular non-covalent coordinate. After bond formation the oscillation amplitude decreases over time as a result of the relaxation of the Diels-Alder adduct.

The applicability of GOSTSHYP in AIMD simulations is an important feature of the method, since it allows investigations of the interplay between pressure and temperature in a time-resolved manner. This can be expected to be particularly useful when investigating spin crossover processes, in which the temperature required for the spin crossover is pressuredependent. ${ }^{85,86}$

Another illustrative example of the capabilities of GOSTSHYP in the simulation of pressure-induced chemical reactions is the cyclotrimerization of acetylene under pressure yielding benzene. ${ }^{87,88}$ Three acetylene molecules were placed in the same plane to mimic the configuration on the surface of a heterogeneous catalyst. Geometry optimizations of this arrangement of molecules at different pressures were carried out at the B3LYP ${ }^{76-78}$ D3BJ ${ }^{89} / 6-31 G(d)^{79}$ level of theory. The optimization results for a pressure range from 0 to $100 \mathrm{GPa}$ are shown in Figure 8. Indeed, the pressure exerted on the individual acetylene molecules through GOSTSHYP ultimately leads to the formation of benzene at a pressure of $68 \mathrm{GPa}$. The average bond length of newly formed carbon-carbon bonds (cf. Fig. 8) steadily decreases up to $67 \mathrm{GPa}$. At $68 \mathrm{GPa}$ and higher pressures, the carbon-carbon bond 


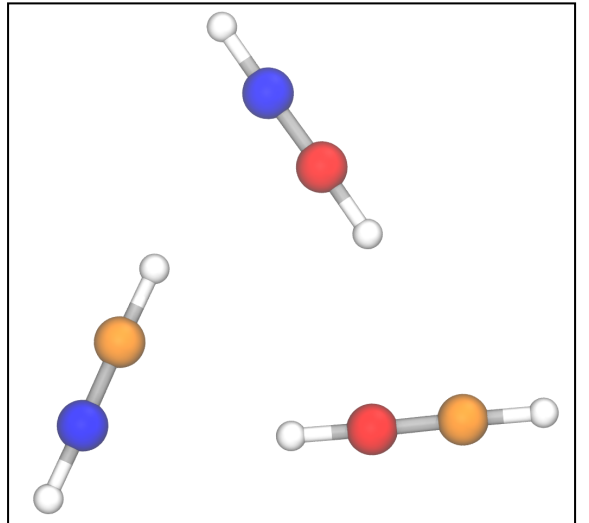

vacuum

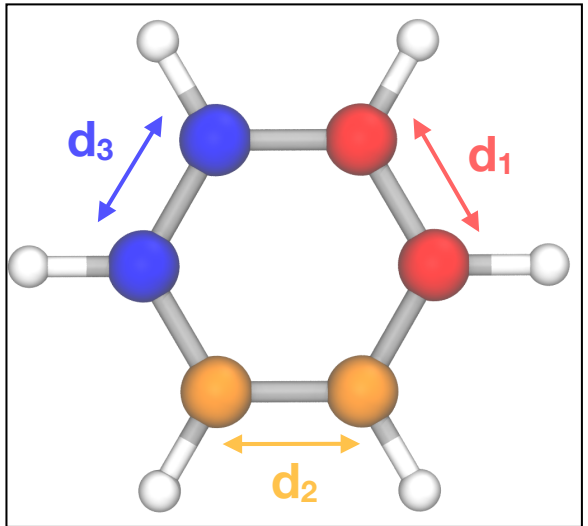

$100 \mathrm{GPa}$

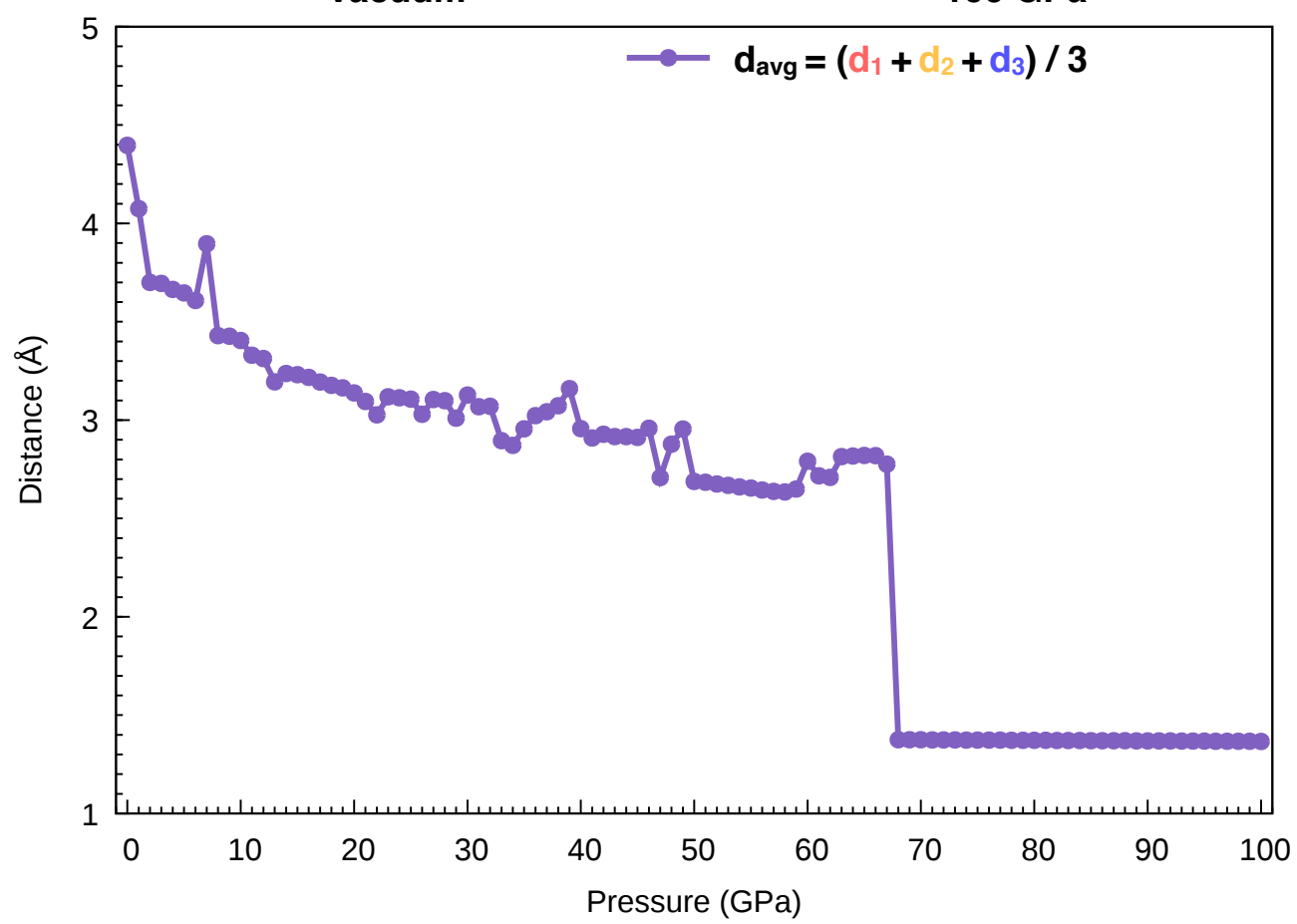

Figure 8: Pressure-induced cyclotrimerization of three acetylene molecules to benzene. The upper panels show the vacuum-optimized geometry with three distinct acetylene molecules and the GOSTSHYP geometry at a pressure of $100 \mathrm{GPa}$. Carbon atoms involved in newly formed bonds are equally colored. The lower panel shows the average bond distances $\mathrm{d}_{\text {avg }}$ of the newly formed bonds at different pressures.

lengths remain at an almost constant value of $1.37 \AA$. Such high pressures are impossible to reach experimentally in the gas phase, where a catalyst is used to obtain benzene out of acetylene. ${ }^{87}$ 


\subsection{Strain Analysis and Compressibility of Small Fullerenes}

The behavior of fullerenes under pressure has been investigated in some detail, ${ }^{90}$ albeit the focus of past studies has been laid on $\mathrm{C}_{60}$. Here, we provide insight into the strain distribution and compressibility of small fullerenes $\left(\mathrm{C}_{20}, \mathrm{C}_{24}, \mathrm{C}_{28}, \mathrm{C}_{32}, \mathrm{C}_{36}, \mathrm{C}_{40}\right)$ due to pressure by using the GOSTSHYP methodology at the B3LYP ${ }^{76-78} / 6-31 \mathrm{G}(\mathrm{d})^{79}$ level of theory and using 110 tessellation points per atom. The initial structures used for the compression simulations are given in the Supporting Information. The connection between pressure and force allows to discuss the compression of molecules in terms of mechanochemistry. ${ }^{47}$ In particular, we apply the Judgement of Energy DIstribution (JEDI) ${ }^{91-93}$ analysis, which quantifies the distribution of strain in a deformed molecule among its bonds, bendings and dihedral angles.

Applying the JEDI analysis for each investigated fullerene at a hydrostatic pressure of $50 \mathrm{GPa}$, one observes that the distributions of strain energy vary significantly from one case to the other (Figure 9a). This is a result of the different symmetries displayed by the fullerenes and the resulting variations in the susceptibilities of different parts of the molecules to compression. In past studies, the localization of strain in inherently strained molecules has been associated with reactivity in the most strained regions of these molecules. ${ }^{94}$ Based on these findings, educated guesses for the pressure-induced reactivity of the investigated fullerenes can now be made, since the regions with highest strain have been identified using the JEDI analysis.

Using again the methodology outlined in Section 3, the volumes of the investigated fullerenes as a function of pressure was calculated (Figure 9b). Considering the ratio in volume at a given pressure and the volume at $P=0 \mathrm{GPa}$, surprisingly, the smallest investigated fullerenes $\left(\mathrm{C}_{20}\right.$ through $\left.\mathrm{C}_{32}\right)$ are more compressible than the larger fullerenes $\mathrm{C}_{36}$ and $\mathrm{C}_{40}$. Intuitively, larger molecules are expected to be more compressible due to their more extended nuclear scaffolds and electron densities, while the opposite is found in the fullerenes considered here. A possible explanation is an increase in stability of larger fullerenes, leading to a reduction in compressibility. While the observed differences are small, further investi- 


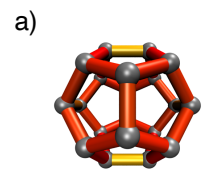

$\mathrm{C}_{20}$

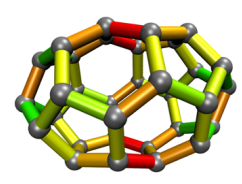

$\mathrm{C}_{32}$

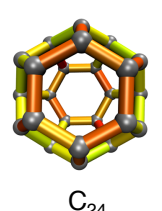

$\mathrm{C}_{24}$

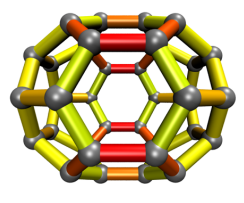

$\mathrm{C}_{36}$
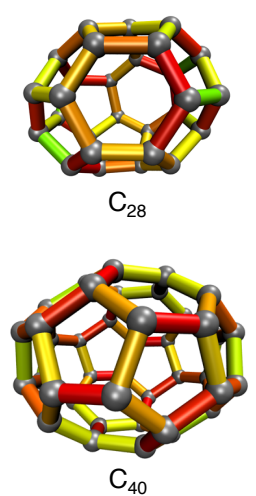

b)

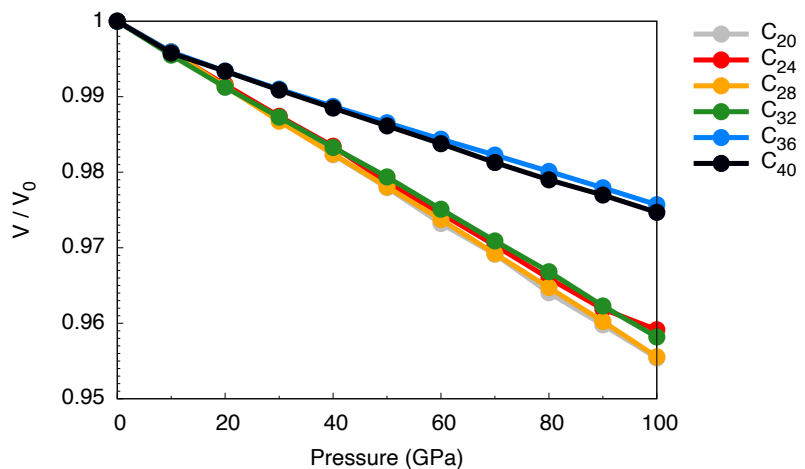

Figure 9: a) Color-coded representation of strain energies in the bonds, bendings and dihedral angles of small fullerenes under pressures of $50 \mathrm{GPa}$, as calculated with the Judgement of Energy DIstribution (JEDI) ${ }^{91-93}$ analysis at the B3LYP ${ }^{76-78} / 6-31 \mathrm{G}(\mathrm{d})^{79}$ level of theory. Strain energies in all internal coordinates of the molecules were mapped onto the covalent bonds. Red signifies high strain, while green represents low strain. Transitions are fluent. b) Ratio of the volume $V$ at a given pressure and the volume $V_{0}$ at $P=0 \mathrm{GPa}$ of the investigated fullerenes as a function of pressure.

gations on a larger set of fullerenes, including different symmetries for fullerenes with the same number of carbon atoms, will need to be carried out to test how general this trend is. The raw volumes of the investigated fullerenes under pressure are given in the Supporting Information (Table S2).

\section{Conclusion and Outlook}

In this paper, an electronic structure method for geometry optimizations and AIMD simulations of chemical systems under pressure, the Gaussians On Surface Tesserae Simulate HYdrostatic Pressure (GOSTSHYP) approach, was introduced. Unlike other established methods for the simulation of atoms and molecules under pressure, the user-defined pressure is applied during geometry optimizations and AIMD simulations, and the compression of electron density is described realistically, as evidenced by physically reasonable pressure-induced changes in electronic energies, volumes and dipole moments calculated with GOSTSHYP. Calculated structural parameters under pressure fall within the range of established methods for computational high-pressure chemistry. GOSTSHYP reproduces the pressure-induced 
Diels-Alder reaction between cyclopentadiene and ethylene, and the cyclotrimierization of acetylene to benzene under pressure is observed. The dependence of the results on the only adjustable parameters of GOSTSHYP (the number of tessellation points per atom and the tessellation sphere scaling factor) is found to be small, which makes it easy to use the method in a black-box manner for geometry optimizations and AIMD simulations of molecules under a desired pressure. GOSTSHYP was implemented in the Q-Chem program package at the SCF level, enabling Hartree-Fock and DFT calculations, and will be made available in a future release of Q-Chem.

In the future, it is planned to use GOSTSHYP for the simulation of metal centers under pressure and test the applicability of the method to reproduce pressure-induced spin crossover processes ${ }^{85,86,95}$ which will contribute to the development of novel storage materials for gases, energy and memory. It will be particularly interesting to compare the performances of GOSTSHYP and mechanochemical models of pressure,${ }^{46,51,52}$ since GOSTSHYP is expected to describe pressure-induced changes in electronic configuration more accurately due to the explicit description of electron density compression. Moreover, a wider range of pressure-induced chemical reactions will be tested with GOSTSHYP in the future with the aim of suggesting new synthetic routes in organic chemistry and beyond. The implementation of an efficient screening algorithm that avoids the calculation of the interaction between a given basis function and remote Gaussian potentials that do not contribute to the energy and the gradient will significantly speed up calculations on larger molecular and supramolecular systems. Another plan focuses on the extension of the GOSTSHYP formalism to electronically excited states by including the compression of electron density due to the Gaussian potentials via a response formalism. This will allow the calculation of UV/Vis absorption spectra under pressure. ${ }^{10,96-98}$ Furthermore, extending the GOSTSHYP formalism beyond the SCF level will allow benchmarking studies of various electronic structure methods, including the Møller-Plesset perturbation theory ${ }^{99}$ and Coupled Cluster ${ }^{100}$ family of methods, in the realm of high-pressure chemistry. Finally, GOSTSHYP will be applied 
to study molecular clusters, e.g. molecules with a solvation shell, to reproduce experiments in which pressure is mediated to solvated molecules via a liquid medium. ${ }^{7,8}$ Interfacing GOSTSHYP with implicit solvent models will be an important intermediate step in this direction.

\section{Acknowledgement}

This work was supported by the Deutsche Forschungsgemeinschaft (DFG) by means of the research training group "CLiC" (GRK 1986, Complex Light Control) as well as the grants STA 1526/1-1 and STA 1526/2-1. MHG acknowledges support from the Director, Office of Science, Office of Basic Energy Sciences, of the U.S. Department of Energy under Contract No. DE-AC02-05CH11231.

\section{Supporting Information Available}

Dipole moments and structural data of water under pressure; volumes of fullerenes under pressure; initial geometries used in the Diels-Alder reaction between cyclopentadiene and ethylene, the cyclotrimerization of acetylene and the strain analysis of the fullerenes; movie of a representative Born-Oppenheimer Molecular Dynamics simulation of the Diels-Alder reaction between cyclopentadiene and ethylene 


\section{A Analytical Nuclear Gradients}

The derivation of analytical gradients with respect to nuclear coordinate displacement in the GOSTSHYP model are outlined in the following. A short-hand notation for integrals according to $\left\langle\phi_{p}\left|G_{j}\right| \phi_{q}\right\rangle=\left\langle p\left|G_{j}\right| q\right\rangle$ will be used. Recall the definition of the Fock operator in the presence of the Gaussian potentials,

$$
\begin{aligned}
F_{p q}= & h_{p q}+\sum_{r s} D_{r s}(\langle p r \mid q s\rangle-\langle p r \mid s q\rangle) \\
& +\sum_{j}\left[\left\langle p\left|G_{j}\right| q\right\rangle-P_{\text {inp }} A_{j}\left[\sum_{r s} D_{r s}\left\langle r\left|\tilde{G}_{j}\right| s\right\rangle \mathcal{F}_{j, p q}\left(\sum_{r s} D_{r s} \mathcal{F}_{j, r s}\right)^{-2}\right]\right]
\end{aligned}
$$

with the matrix elements $h_{p q}$ of the core Hamiltonian and the two-electron integrals in physicists' notation $\langle p r \mid q s\rangle .{ }^{101}$ Let

$$
E_{\mathrm{SCF}}=\sum_{p q} D_{p q} h_{p q}+\frac{1}{2} \sum_{p q r s} D_{p q} D_{r s}(\langle p r \mid q s\rangle-\langle p r \mid s q\rangle)+V_{\mathrm{nn}}+\sum_{j} \sum_{p q} D_{p q}\left\langle p\left|G_{j}\right| q\right\rangle
$$

be the total SCF energy in presence of Gaussian potentials. Here, $V_{n n}$ is the nuclear repulsion energy. Now consider the derivative of the SCF energy with respect to a general perturbation $\lambda,{ }^{101,102}$

$$
\begin{aligned}
\frac{\partial E_{\mathrm{SCF}}}{\partial \lambda}= & \sum_{p q} \frac{\partial D_{p q}}{\partial \lambda} h_{p q}+\sum_{p q} D_{p q} \frac{\partial h_{p q}}{\partial \lambda}+\frac{1}{2} \sum_{p q r s}\left(\frac{\partial D_{p q}}{\partial \lambda} D_{r s}+D_{p q} \frac{\partial D_{r s}}{\partial \lambda}\right)(\langle p r \mid q s\rangle-\langle p r \mid s q\rangle) \\
& +\frac{1}{2} \sum_{p q r s} D_{p q} D_{r s} \frac{\partial}{\partial \lambda}(\langle p r \mid q s\rangle-\langle p r \mid s q\rangle)+\frac{\partial V_{\mathrm{nn}}}{\partial \lambda} \\
& +\sum_{j} \frac{\partial}{\partial \lambda} P_{\mathrm{inp}} A_{j} \sum_{p q} D_{p q}\left\langle p\left|\tilde{G}_{j}\right| q\right\rangle\left(\sum_{p q} D_{p q} \mathcal{F}_{j, p q}\right)^{-1}
\end{aligned}
$$


Everything but the last term is identical to the vacuum analytical SCF energy gradient, so only the final term is now considered separately for each tessellation point $j$, i.e.,

$$
\begin{aligned}
E_{\mathrm{p}, j}^{\lambda}= & \frac{\partial}{\partial \lambda} P_{\mathrm{inp}} A_{j} \sum_{p q} D_{p q}\left\langle p\left|\tilde{G}_{j}\right| q\right\rangle\left(\sum_{p q} D_{p q} \mathcal{F}_{j, p q}\right)^{-1} \\
= & \underbrace{P_{\mathrm{inp}} \frac{\partial A_{j}}{\partial \lambda} \sum_{p q} D_{p q}\left\langle p\left|\tilde{G}_{j}\right| q\right\rangle\left(\sum_{p q} D_{p q} \mathcal{F}_{j, p q}\right)^{-1}}_{E_{\mathrm{p}, j}^{\lambda,(1)}} \\
& +\underbrace{P_{\mathrm{inp}} A_{j}\left(\sum_{p q} D_{p q} \mathcal{F}_{j, p q}\right)^{-1}\left(\frac{\partial}{\partial \lambda} \sum_{p q} D_{p q}\left\langle p\left|\tilde{G}_{j}\right| q\right\rangle\right)}_{E_{\mathrm{p}, j}^{\lambda,(2)}} \\
& +\underbrace{P_{\mathrm{inp}} A_{j} \sum_{p q} D_{p q}\left\langle p\left|\tilde{G}_{j}\right| q\right\rangle \frac{\partial}{\partial \lambda}\left(\sum_{p q} D_{p q} \mathcal{F}_{j, p q}\right)^{-1}}_{E_{\mathrm{p}, j}^{\lambda,(3)}} .
\end{aligned}
$$

The first term in eq (A5) contains the cavitiy derivative, ${ }^{103,104}$ which can be computed with the present routines for the non-electrostatic PCM nuclear gradients. Carrying out the derivatives in the second term, $E_{\mathrm{p}, j}^{\lambda,(2)}$, yields

$$
\begin{aligned}
E_{\mathrm{p}, j}^{\lambda,(2)} & =p_{j} \sum_{p q} D_{p q} \frac{\partial\left\langle p\left|\tilde{G}_{j}\right| q\right\rangle}{\partial \lambda} \\
& =p_{j} \sum_{p q} D_{p q}\left(\left\langle\frac{\partial p}{\partial \lambda}\left|\tilde{G}_{j}\right| q\right\rangle+\left\langle p\left|\tilde{G}_{j}\right| \frac{\partial q}{\partial \lambda}\right\rangle+\left\langle p\left|\frac{\partial \tilde{G}_{j}}{\partial \lambda}\right| q\right\rangle\right)
\end{aligned}
$$

Note that all derivatives of the one-particle density matrix are already consumed into the energy-weighted density matrix contracted with the derivatives of the overlap matrix, ${ }^{102}$ such that we only need to consider the derivative of the operator explicitly. Hence, the last term of equation (A7) requires further attention,

$$
\left\langle p\left|\frac{\partial \tilde{G}_{j}}{\partial \lambda}\right| q\right\rangle=-\frac{\partial w_{j}}{\partial \lambda}\left\langle p\left|\left(\boldsymbol{r}-\boldsymbol{r}_{j}\right)^{2} \tilde{G}_{j}\right| q\right\rangle-w_{j}\left\langle p\left|\frac{\partial}{\partial \lambda}\left(\boldsymbol{r}-\boldsymbol{r}_{j}\right)^{2} \tilde{G}_{j}\right| q\right\rangle,
$$


where the first term contains a derivative of the width parameter $w_{j}$ multiplied with a three-center overlap integral with a d-type function on the mid center, and the second term represents the Hellmann-Feynman force through the Gaussian potential. The derivative of the width parameter is straightforward since it depends on the cavity derivative given by

$$
\begin{aligned}
\frac{\partial w_{j}}{\partial \lambda} & =\frac{\partial}{\partial \lambda} \frac{\pi \ln 2}{A_{j}} \\
& =\pi \ln 2 \frac{\partial}{\partial \lambda} \frac{1}{A_{j}} \\
& =-\pi \ln 2 \frac{\partial A_{j}}{\partial \lambda} \frac{1}{A_{j}^{2}}
\end{aligned}
$$

Considering the last term of eq $(\mathrm{A} 5), E_{\mathrm{p}, j}^{\lambda,(3)}$, one obtains

$$
E_{\mathrm{p}, j}^{\lambda,(3)}=-P_{\mathrm{inp}} A_{j}\left(\sum_{r s} D_{r s}\left\langle r\left|\tilde{G}_{j}\right| s\right\rangle\right)\left(\sum_{r s} D_{r s} \mathcal{F}_{j, r s}\right)^{-2} \sum_{p q} D_{p q} \frac{\partial}{\partial \lambda} \mathcal{F}_{j, p q}
$$

The derivative of $\mathcal{F}_{j, p q}$ is expanded to

$$
\begin{aligned}
\frac{\partial}{\partial \lambda} \mathcal{F}_{j, p q}=\frac{\partial}{\partial \lambda}( & n_{x}\left\langle p\left|2 w_{j}\left(x-x_{j}\right) \exp \left(-w_{j}\left(\boldsymbol{r}-\boldsymbol{r}_{j}\right)^{2}\right)\right| q\right\rangle \\
& +n_{y}\left\langle p\left|2 w_{j}\left(y-y_{j}\right) \exp \left(-w_{j}\left(\boldsymbol{r}-\boldsymbol{r}_{j}\right)^{2}\right)\right| q\right\rangle \\
& \left.+n_{z}\left\langle p\left|2 w_{j}\left(z-z_{j}\right) \exp \left(-w_{j}\left(\boldsymbol{r}-\boldsymbol{r}_{j}\right)^{2}\right)\right| q\right\rangle\right) .
\end{aligned}
$$

The first term (the two other terms can be treated analogously) yields three different contributions through the derivative, i.e.,

$$
\begin{aligned}
\frac{\partial}{\partial \lambda}\left\langle p\left|2 w_{j}\left(x-x_{j}\right) \exp \left(-w_{j}\left(\boldsymbol{r}-\boldsymbol{r}_{j}\right)^{2}\right)\right| q\right\rangle=n_{x}[ & \left\langle\frac{\partial p}{\partial \lambda}\left|2 w_{j}\left(x-x_{j}\right) \exp \left(-w_{j}\left(\boldsymbol{r}-\boldsymbol{r}_{j}\right)^{2}\right)\right| q\right\rangle \\
& +\left\langle p\left|2 w_{j}\left(x-x_{j}\right) \exp \left(-w_{j}\left(\boldsymbol{r}-\boldsymbol{r}_{j}\right)^{2}\right)\right| \frac{\partial q}{\partial \lambda}\right\rangle \\
& \left.+\left\langle p\left|\frac{\partial}{\partial \lambda} 2 w_{j}\left(x-x_{j}\right) \exp \left(-w_{j}\left(\boldsymbol{r}-\boldsymbol{r}_{j}\right)^{2}\right)\right| q\right\rangle\right],
\end{aligned}
$$


where the first two terms are simple derivatives of a three-center overlap integral on the "bra" and "ket" side, respectively. The last term is more involved due to the non-vanishing derivative of the width parameter,

$$
\begin{aligned}
& \left\langle p\left|\frac{\partial}{\partial \lambda} 2 w_{j}\left(x-x_{j}\right) \exp \left(-w_{j}\left(\boldsymbol{r}-\boldsymbol{r}_{j}\right)^{2}\right)\right| q\right\rangle \\
= & 2 \frac{\partial w_{j}}{\partial \lambda}\left\langle p\left|\left(x-x_{j}\right) \exp \left(-w_{j}\left(\boldsymbol{r}-\boldsymbol{r}_{j}\right)^{2}\right)\right| q\right\rangle \\
& +2 w_{j}\left\langle p\left|\frac{\partial}{\partial \lambda}\left(x-x_{j}\right) \exp \left(-w_{j}\left(\boldsymbol{r}-\boldsymbol{r}_{j}\right)^{2}\right)\right| q\right\rangle \\
= & 2 \frac{\partial w_{j}}{\partial \lambda}\left\langle p\left|\left(x-x_{j}\right) \exp \left(-w_{j}\left(\boldsymbol{r}-\boldsymbol{r}_{j}\right)^{2}\right)\right| q\right\rangle \\
& +2 w_{j}\langle p| \exp \left(-w_{j}\left(\boldsymbol{r}-\boldsymbol{r}_{j}\right)^{2}\right) \\
& \times\left[\frac{\partial\left(x-x_{j}\right)}{\partial \lambda}-2\left(x-x_{j}\right)\left(\boldsymbol{r}-\boldsymbol{r}_{j}\right) \frac{\partial\left(\boldsymbol{r}-\boldsymbol{r}_{j}\right)}{\partial \lambda} w_{j}-\left(x-x_{j}\right)\left(\boldsymbol{r}-\boldsymbol{r}_{j}\right)^{2} \frac{\partial w_{j}}{\partial \lambda}\right]|q\rangle
\end{aligned}
$$

The red terms in the above equations arise from the standard derivative of the Gaussian with perturbation-independent exponents, whereas the blue term gives rise to an overlap integral involving (Cartesian) f-type orbitals on the mid center multiplied with the derivative of the width parameter given in eq (A9). Collecting and assembling the terms for the other two Cartesian components is straightforward. 


\section{References}

(1) Grochala, W.; Hoffmann, R.; Feng, J.; Ashcroft, N. W. The Chemical Imagination at Work in Very Tight Places. Angew. Chem. Int. Ed. 2007, 46, 3620-3642.

(2) Schettino, V.; Bini, R. Constraining molecules at the closest approach: chemistry at high pressure. Chem. Soc. Rev. 2007, 36, 869-880.

(3) McMillan, P. F. Chemistry at high pressure. Chem. Soc. Rev. 2006, 35, 855-857.

(4) Li, B.; Ji, C.; Yang, W.; Wang, J.; Yang, K.; Xu, R.; Liu, W.; Cai, Z.; Chen, J.; kwang Mao, H. Diamond anvil cell behavior up to 4 Mbar. Proc. Nat. Acad. Sci. U.S.A. 2018, $115,1713-1717$.

(5) Asay, J. R., Shahinpoor, M., Eds. High-Pressure Shock Compression of Solids, 1st ed.; Springer Science+Business Media: New York, 1993.

(6) Casati, N.; Kleppe, A.; Jephcoat, A. P.; Macchi, P. Putting pressure on aromaticity along with in situ experimental electron density of a molecular crystal. Nat. Commun. 2016, ๆ, 10901.

(7) Chandrasekhar, M.; Guha, S.; Graupner, W. Squeezing Organic Conjugated Molecules - What Does One Learn? Adv. Mater. 2001, 13, 613-618.

(8) Kato, M.; Higashi, M.; Taniguchi, Y. Effect of pressure on the internal rotation angle of biphenyl in carbon disulfide. J. Chem. Phys. 1988, 89, 5417-5421.

(9) Brown, I. D.; Klages, P.; Skowron, A. Influence of pressure on the lengths of chemical bonds. Acta Crystallogr. 2003, B59, 439-448.

(10) Puschnig, P.; Ambrosch-Draxl, C.; Heimel, G.; Zojer, E.; Resel, R.; Leising, G.; Kriechbaum, M.; Graupner, W. Pressure studies on the intermolecular interactions in biphenyl. Synth. Met. 2001, 116, 327-331. 
(11) Katrusiak, A. High-Pressure X-Ray Diffraction Study Of 2-Methyl-1,3Cyclopentanedione Crystals. High Press. Res. 1991, 6, 155-167.

(12) Cartz, L.; Srinivasa, S. R.; Riedner, R. J.; Jorgensen, J. D.; Worlton, T. G. Effect of pressure on bonding in black phosphorus. J. Chem. Phys. 1979, 71, 1718-1721.

(13) Klärner, F.-G. Chemie unter Hochdruck. Chemie Unserer Zeit 1989, 23, 53-63.

(14) Stauch, T. Quantum chemical modeling of molecules under pressure. Int. J. Quantum Chem. 2020, e26208.

(15) Pašteka, L. F.; Helgaker, T.; Saue, T.; Sundholm, D.; Werner, H.-J.; Hasanbulli, M.; Major, J.; Schwerdtfeger, P. Atoms and molecules in soft confinement potentials. Mol. Phys. 2020, DOI: 10.1080/00268976.2020.1730989.

(16) Novoa, T.; Contreras-García, J.; Fuentealba, P.; Cárdenas, C. The Pauli principle and the confinement of electron pairs in a double well: Aspects of electronic bonding under pressure. J. Chem. Phys. 2019, 150, 204304.

(17) Aquino, N. The Hydrogen and Helium Atoms Confined in Spherical Boxes. Adv. Quantum Chem. 2009, 57, 123-171.

(18) Gorecki, J.; Byers Brown, W. On the ground state of the hydrogen molecule-ion H2+ enclosed in hard and soft spherical boxes. J. Chem. Phys. 1988, 89, 2138-2148.

(19) Weisskopf, V. F. Search for Simplicity: Quantum mechanics and the Pauli principle. Am. J. Physiol. 1985, 53, 109-110.

(20) LeSar, R.; Herschbach, D. R. Electronic and Vibrational Properties of Molecules at High Pressures. Hydrogen Molecule in a Rigid Spheroidal Box. J. Chem. Phys. 1981, 85, 2798-2804.

(21) Ley-Koo, E.; Rubinstein, S. The hydrogen atom within spherical boxes with penetrable walls. J. Chem. Phys. 1979, 71, 351-357. 
(22) Ludeña, E. V. SCF calculations for hydrogen in a spherical box. J. Chem. Phys. 1977, $66,468-470$.

(23) Suryanarayana, D.; Weil, J. A. On the hyperfine splitting of the hydrogen atom in a spherical box. J. Chem. Phys. 1976, 64, 510-513.

(24) Michels, A.; De Boer, J.; Bijl, A. Remarks Concerning Molecular Interaction and their Influence on the Polarisability. Physica 1937, 4, 981-994.

(25) Miao, M.-S. Caesium in high oxidation states and as a p-block element. Nat. Chem. 2013, 5, 846-852.

(26) Selli, D.; Baburin, I. A.; Martoňák, R.; Leoni, S. Novel metastable metallic and semiconducting germaniums. Sci. Rep. 2013, 3, 1466.

(27) Huan, T. D.; Amsler, M.; Marques, M. A. L.; Botti, S.; Willand, A.; Goedecker, S. Low-Energy Polymeric Phases of Alanates. Phys. Rev. Lett. 2013, 110, 135502.

(28) Wang, H.; Tse, J. S.; Tanaka, K.; Iitaka, T.; Ma, Y. Superconductive sodalite-like clathrate calcium hydride at high pressures. Proc. Nat. Acad. Sci. U.S.A. 2012, 109, 6463-6466.

(29) Amsler, M. et al. Crystal Structure of Cold Compressed Graphite. Phys. Rev. Lett. 2012, 108, 065501.

(30) Flores-Livas, J. A.; Amsler, M.; Lenosky, T. J.; Lehtovaara, L.; Botti, S.; Marques, M. A. L.; Goedecker, S. High-Pressure Structures of Disilane and Their Superconducting Properties. Phys. Rev. Lett. 2012, 108, 117004.

(31) Kurzydłowskia, D.; Grochala, W. Phonon Dispersion Analysis as an Indispensable Tool for Predictions of Solid State Polymorphism and Dynamic Metastability: Case of Compressed Silane D. Acta Phys. Pol. A 2011, 119, 895-900. 
(32) Yao, Y.; Tse, J. S.; Klug, D. D. Structures of Insulating Phases of Dense Lithium. Phys. Rev. Lett. 2009, 102, 115503.

(33) Zurek, E.; Grochala, W. Predicting crystal structures and properties of matter under extreme conditions via quantum mechanics: The pressure is on. Phys. Chem. Chem. Phys. 2015, 17, 2917-2934.

(34) Nosé, S.; Klein, M. L. Constant pressure molecular dynamics for molecular systems. Mol. Phys. 1983, 50, 1055-1076.

(35) Demontis, P.; Lesar, R.; Klein, M. L. New High-Pressure Phases of Ice. Phys. Rev. Lett. 1988, 60, 2284-2287.

(36) Kitchen, D. B.; Reed, L. H.; Levy, R. M. Molecular Dynamics Simulation of Solvated Protein at High Pressure. Biochemistry 1992, 31, 10083-10093.

(37) Tarnacka, M. et al. Molecular dynamics of itraconazole at ambient and high pressure. Phys. Chem. Chem. Phys. 2013, 15, 20742-20752.

(38) Horbach, J. Molecular dynamics computer simulation of amorphous silica under high pressure. J. Phys.: Condens. Matter 2008, 20, 244118.

(39) Rustad, J. R.; Yuen, D. A.; Spera, F. J. Molecular dynamics of liquid SiO2 under high pressure. Phys. Rev. A 1990, 42, 2081-2089.

(40) Zeidler, A. et al. High-Pressure Transformation of $\mathrm{SiO} 2$ Glass from a Tetrahedral to an Octahedral Network: A Joint Approach Using Neutron Diffraction and Molecular Dynamics. Phys. Rev. Lett. 2014, 113, 135501.

(41) Marx, D.; Hutter, J. Ab Initio Molecular Dynamics. Basic Theory and Advanced Methods, 1st ed.; Cambridge University Press: Cambridge, UK, 2009.

(42) Attaccalite, C.; Sorella, S. Stable Liquid Hydrogen at High Pressure by a Novel Ab Initio Molecular-Dynamics Calculation. Phys. Rev. Lett. 2008, 100, 114501. 
(43) Mugnai, M.; Cardini, G.; Schettino, V. Charge separation and polymerization of hydrocarbons at an ultrahigh pressure. Phys. Rev. B 2004, 70, 020101.

(44) Imoto, S.; Marx, D. How Can Protons Migrate in Extremely Compressed Liquid Water? Phys. Rev. Lett. 2020, 125, 086001.

(45) Imoto, S.; Kibies, P.; Rosin, C.; Winter, R.; Kast, S. M.; Marx, D. Toward Extreme Biophysics: Deciphering the Infrared Response of Biomolecular Solutions at High Pressures. Angew. Chem. Int. Ed. 2016, 55, 9534-9538.

(46) Subramanian, G.; Mathew, N.; Leiding, J. A generalized force-modified potential energy surface for mechanochemical simulations. J. Chem. Phys. 2015, 143, 134109.

(47) Stauch, T.; Dreuw, A. Advances in Quantum Mechanochemistry: Electronic Structure Methods and Force Analysis. Chem. Rev. 2016, 116, 14137-14180.

(48) Ong, M. T.; Leiding, J.; Tao, H.; Virshup, A. M.; Martínez, T. J. First Principles Dynamics and Minimum Energy Pathways for Mechanochemical Ring Opening of Cyclobutene. J. Am. Chem. Soc. 2009, 131, 6377-6379.

(49) Jha, S. K.; Brown, K.; Todde, G.; Subramanian, G. A mechanochemical study of the effects of compression on a Diels-Alder reaction. J. Chem. Phys. 2016, 145, 074307.

(50) Todde, G.; Jha, S. K.; Subramanian, G. The effect of external forces on the initial dissociation of RDX (1,3,5-trinitro-1,3,5-triazine): A mechanochemical study. Int. J. Quantum Chem. 2017, 117, e25426.

(51) Stauch, T.; Chakraborty, R.; Head-Gordon, M. Quantum Chemical Modeling of Pressure-Induced Spin Crossover in Octahedral Metal-Ligand Complexes. ChemPhysChem 2019, 20, 2742-2747.

(52) Stauch, T. A mechanochemical model for the simulation of molecules and molecular crystals under hydrostatic pressure. J. Chem. Phys. 2020, 153, 134503. 
(53) Cammi, R.; Verdolino, V.; Mennucci, B.; Tomasi, J. Towards the elaboration of a QM method to describe molecular solutes under the effect of a very high pressure. Chem. Phys. 2008, 344, 135-141.

(54) Cammi, R. A New Extension of the Polarizable Continuum Model: Toward a Quantum Chemical Description of Chemical Reactions at Extreme High Pressure. J. Comput. Chem. 2015, 36, 2246-2259.

(55) Chen, B.; Hoffmann, R.; Cammi, R. The Effect of Pressure on Organic Reactions in Fluids - a New Theoretical Perspective. Angew. Chem. Int. Ed. 2017, 56, 11126-11142.

(56) Fukuda, R.; Nakatani, K. Quantum Chemical Study on the High-Pressure Effect for $[4+4]$ Retrocycloaddition of Anthracene Cyclophane Photodimer. J. Phys. Chem. C 2019, 123, 4493-4501.

(57) Pagliai, M.; Cardini, G.; Cammi, R. Vibrational Frequencies of Fullerenes C60 and C70 under Pressure Studied with a Quantum Chemical Model Including Spatial Confinement Effect. J. Phys. Chem. A 2014, 118, 5098-5111.

(58) Fukuda, R.; Ehara, M.; Cammi, R. Modeling molecular systems at extreme pressure by an extension of the polarizable continuum model (PCM) based on the symmetryadapted cluster-configuration interaction (SAC-CI) method: Confined electronic excited states of furan as a test case. J. Chem. Theory Comput. 2015, 11, 2063-2076.

(59) Rahm, M.; Cammi, R.; Ashcroft, N. W.; Hoffmann, R. Squeezing All Elements in the Periodic Table: Electron Configuration and Electronegativity of the Atoms under Compression. J. Am. Chem. Soc. 2019, 141, 10254-10271.

(60) Caratelli, C.; Cammi, R.; Chelli, R.; Pagliai, M.; Cardini, G.; Schettino, V. Insights on the Realgar Crystal under Pressure from XP-PCM and Periodic Model Calculations. J. Phys. Chem. A 2017, 121, 8825-8834. 
(61) Cammi, R.; Cappelli, C.; Mennucci, B.; Tomasi, J. Calculation and analysis of the harmonic vibrational frequencies in molecules at extreme pressure: Methodology and diborane as a test case. J. Chem. Phys. 2012, 137, 154112.

(62) Shao, Y. et al. Advances in molecular quantum chemistry contained in the Q-Chem 4 program package. Mol. Phys. 2014, 113, 184-215.

(63) Hartree, D. R. The Wave Mechanics of an Atom with a Non-Coulomb Central Field. Math. Proc. Camb. Philos. Soc. 1928, 24, 111-132.

(64) Hartree, D. R.; Hartree, W. Self-Consistent Field, with Exchange, for Beryllium. Proc. Royal Soc. Lond. A 1935, 150, 9-33.

(65) Hohenberg, P.; Kohn, W. Inhomogeneous Electron Gas. Phys. Rev. 1964, 136, 864871.

(66) Kohn, W.; Sham, L. J. Self-Consistent Equations Including Exchange and Correlation Effects. Phys. Rev. 1965, 140, 1133-1138.

(67) Khaliullin, R. Z.; Cobar, E. A.; Lochan, R. C.; Bell, A. T.; Head-Gordon, M. Unravelling the Origin of Intermolecular Interactions Using Absolutely Localized Molecular Orbitals. J. Phys. Chem. A 2007, 111, 8753-8765.

(68) von Hopffgarten, M.; Frenking, G. Energy decomposition analysis. WIREs Comput. Mol. Sci. 2012, 2, 43-62.

(69) Horn, P. R.; Mao, Y.; Head-Gordon, M. Probing non-covalent interactions with a second generation energy decomposition analysis using absolutely localized molecular orbitals. Phys. Chem. Chem. Phys. 2016, 18, 23067-23079.

(70) Lange, A. W.; Herbert, J. M. Polarizable Continuum Reaction-Field Solvation Models Affording Smooth Potential Energy Surfaces. J. Phys. Chem. Lett. 2010, 1, 556-561. 
(71) Rahm, M.; Angqvist, M.; Rahm, J. M.; Erhart, P.; Cammi, R. Non-bonded Radii of the Atoms Under Compression. ChemPhysChem 2020, DOI: 10.1002/cphc.202000624.

(72) Obara, S.; Saika, A. Efficient recursive computation of molecular integrals over Cartesian Gaussian functions. J. Chem. Phys. 1986, 84, 3963-3974.

(73) Wolfram, Mathematica, Version 12.0. https://www.wolfram.com/mathematica.

(74) Bondi, A. van der Waals Volumes and Radii. J. Phys. Chem. 1964, 68, 441-451.

(75) Wong, M. W.; Wiberg, K. B.; Frisch, M. J. Ab lnitio Calculation of Molar Volumes: Comparison with Experiment and Use in Solvation Models. J. Comput. Chem. 1995, $16,385-394$.

(76) Becke, A. D. Density-functional exchange-energy approximation with correct asymptotic behavior. Phys. Rev. A 1988, 38, 3098-3100.

(77) Lee, C.; Yang, W.; Parr, R. G. Development of the Colle-Salvetti correlation-energy formula into a functional of the electron density. Phys. Rev. B 1988, 37, 785-789.

(78) Becke, A. D. A new mixing of Hartree-Fock and local density-functional theories. $J$. Chem. Phys. 1993, 98, 1372-1377.

(79) Hehre, W. J.; Ditchfield, R.; Pople, J. A. Self-Consistent Molecular Orbital Methods. XII. Further Extensions of Gaussian-Type Basis Sets for Use in Molecular Orbital Studies of Organic Molecules. J. Chem. Phys. 1972, 56, 2257-2261.

(80) Dunning, T. H. Gaussian basis sets for use in correlated molecular calculations. I. The atoms boron through neon and hydrogen. J. Chem. Phys. 1989, 90, 1007-1023.

(81) Zhao, Y.; Truhlar, D. G. The M06 suite of density functionals for main group thermochemistry, thermochemical kinetics, noncovalent interactions, excited states, and transition elements: Two new functionals and systematic testing of four M06-class functionals and 12 other function. Theor. Chem. Acc. 2008, 120, 215-241. 
(82) Cruz, S. A.; Soullard, J. Pressure effects on the electronic and structural properties of molecules. Chem. Phys. Lett. 2004, 391, 138-142.

(83) Kang, D.; Dai, J.; Yuan, J. Changes of structure and dipole moment of water with temperature and pressure: A first principles study. J. Chem. Phys. 2011, 135, 024505.

(84) Perdew, J. P.; Burke, K.; Ernzerhof, M. Generalized Gradient Approximation Made Simple. Phys. Rev. Lett. 1996, 77, 3865-3868.

(85) Ksenofontov, V.; Gaspar, A. B.; Gütlich, P. Pressure Effect Studies on Spin Crossover and Valence Tautomeric Systems. Top. Curr. Chem. 2004, 235, 23-64.

(86) Gütlich, P.; Ksenofontov, V.; Gaspar, A. B. Pressure effect studies on spin crossover systems. Coord. Chem. Rev. 2005, 249, 1811-1829.

(87) Vollhardt, K. P. C. Transition-Metal-Catalyzed Acetylene Cyclizations in Organic Synthesis. Acc. Chem. Res. 1977, 10, 1-8.

(88) Breitkopf, V.; Hopf, H.; Klärner, F.; Witulski, B.; Zimny, B. The Effect of Pressure on the Trimerization and Diels-Alder Reaction of Cyanoacetylene. Synthesis and Reactivity of 2,3,5-Tricyanobicyclo(2.2.0]hexa-2,5-diene ("Tricyano Dewar Benzene"). Liebigs Ann. 1995, 613-617.

(89) Grimme, S.; Antony, J.; Ehrlich, S.; Krieg, H. A consistent and accurate ab initio parametrization of density functional dispersion correction (DFT-D) for the 94 elements H-Pu. J. Chem. Phys. 2010, 132, 154104.

(90) Sundqvist, B. Fullerenes under high pressures. Adv. Phys. 1999, 48, 1-134.

(91) Stauch, T.; Dreuw, A. A quantitative quantum-chemical analysis tool for the distribution of mechanical force in molecules. J. Chem. Phys. 2014, 140, 134107.

(92) Stauch, T.; Dreuw, A. On the use of different coordinate systems in mechanochemical force analyses. J. Chem. Phys. 2015, 143, 074118. 
(93) Stauch, T.; Dreuw, A. Quantum Chemical Strain Analysis For Mechanochemical Processes. Acc. Chem. Res. 2017, 50, 1041-1048.

(94) Colwell, C. E.; Price, T. W.; Stauch, T.; Jasti, R. Strain Visualization for Strained Macrocycles. Chem. Sci. 2020, 11, 3923-3930.

(95) Zarembowitch, J.; Roux, C.; Boillot, M. L.; Claude, R.; Itie, J. P.; Polian, A.; Bolte, M. Temperature-, Pressure- and Light-Induced Electronic Spin Conversions in Transition Metal Complexes. Mol. Cryst. Liq. Cryst. 1993, 234, 247-254.

(96) Robertson, W. W.; Babb, S. E.; Matsen, F. A. Effects of High Pressure on the Near Ultraviolet Absorption Spectra of Benzene and Monosubstituted Benzenes. The Journal of Chemical Physics 1957, 26, 367-370.

(97) Aust, R. B.; Bentley, W. H.; Drickamer, H. G. Behavior of Fused-Ring Aromatic Hydrocarbons at Very High Pressure. J. Chem. Phys. 1964, 41, 1856-1864.

(98) Neumann, B.; Pollmann, P. Investigation of two cyanine dyes at normal and high pressure by UV/Vis spectroscopy. Phys. Chem. Chem. Phys. 2000, 2, 4784-4792.

(99) Møller, C.; Plesset, M. S. Note on an Approximation Treatment for Many-Electron Systems. Phys. Rev. 1934, 46, 618-622.

(100) Coester, F.; Kümmel, H. Short-Range Correlations in Nuclear Wave Functions. Nucl. Phys. 1960, 17, 477-485.

(101) Jensen, J. H. Modeling intermolecular exchange integrals between nonorthogonal molecular orbitals. J. Chem. Phys. 1996, 104, 7795-7796.

(102) Pulay, P. Ab initio calculation of force constants and equilibrium geometries in polyatomic molecules. Mol. Phys. 1969, 17, 197-204.

(103) Cossi, M.; Mennucci, B.; Cammi, R. Analytical First Derivatives of Molecular Surfaces with Respect to Nuclear Coordinates. J. Comput. Chem. 1996, 17, 57-73. 
(104) Cossi, M.; Scalmani, G.; Rega, N.; Barone, V. New developments in the polarizable continuum model for quantum mechanical and classical calculations on molecules in solution. J. Chem. Phys. 2002, 117, 43-54. 
Graphical TOC Entry

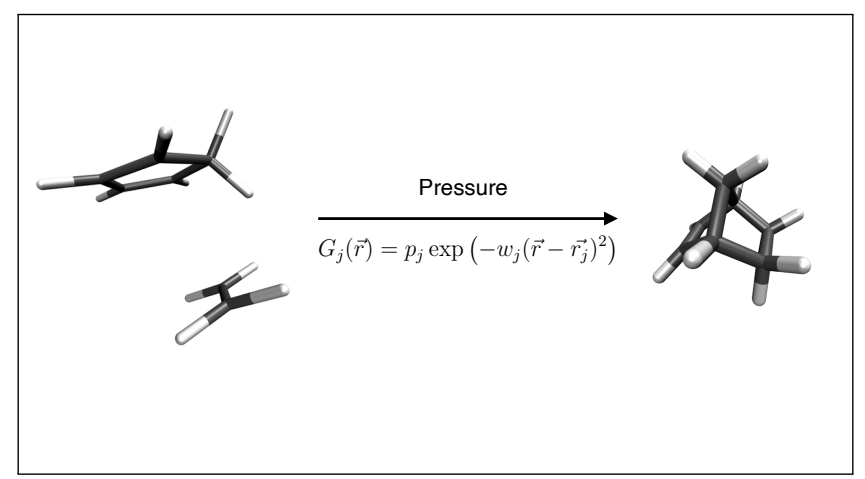

